\title{
Two-To-One Internal Resonance of an Inclined Marine Riser under Harmonic Excitations
}

\author{
Feras K. Alfosail \\ Mohammad I. Younis \\ Physical Sciences and Engineering Division, King Abdullah University of Science \\ and Technology, Thuwal 23955-9600, Saudi Arabia.
}

\begin{abstract}
In this paper, we study the two-to-one internal resonance of an inclined marine riser under harmonic excitations. The riser is modeled as an Euler-Bernoulli beam accounting for mid-plane stretching, self-weight, and an applied axial top tension. Due to the inclination, the self-weight load causes a static deflection of the riser, which can tune the frequency ratio between the third and first natural frequencies near two. The multiple time scales method is applied to study the nonlinear equation accounting for the system nonlinearity. The solution is then compared to a Galerkin solution showing good agreement. A further investigation is carried out by plotting the frequency response curves, the force response curves, and the steady state response of the multiple scales solution, in addition to the dynamical solution obtained by Galerkin, as they vary with the detuning parameters. The results reveal that the riser vibrations can undergo multiple Hopf bifurcations and experience quasi-periodic motion that can lead to chaotic behavior. These phenomena lead to complex vibrations of the riser, which can accelerate its fatigue failure.
\end{abstract}

Keywords: Harmonic excitation, internal resonance, marine riser, two-to-one.

\section{Introduction}

Marine risers are pipe structures designed for transporting oil and gas in drilling and production platforms. The riser structure is subjected to various environmental loads, which include heave top motions, sea currents, and internal fluid flow. In addition, the riser structure inherits nonlinear geometric effects that can cause complex nonlinear dynamics of the beam, such as multi-modal interactions via internal resonance and parametric excitation, which can lead to *Corresponding author: Mohammad I. Younis $(\bowtie)$, Physical Sciences and Engineering Division, King Abdullah University of Science and Technology, Thuwal 23955-9600, Saudi Arabia, e-mail: Mohammad.Younis@kaust.edu.sa, tel.:+966544700154, ORCID: orcid.org/0000-0002-9491-1838

Feras K. Alfosail, Physical Sciences and Engineering Division, King Abdullah University of Science and Technology, Thuwal 23955-9600, Saudi Arabia, email: feras.alfosail@kaust.edu.sa, tel.:+966506847015, ORCID: orcid.org/0000-0002-5553-4945 
chaotic behavior. The combined action of environmental loads and nonlinear geometric effects can result into vortex induced vibration (VIV) phenomena, which hinder the life of the riser by fatigue [1]. Therefore, it is necessary to study and examine the nonlinear response of the riser under these circumstances and understand the nonlinear interaction to prevent the failure of the riser structure.

The riser is often modeled as an Euler-Bernoulli beam with variable axial force due to self-weight. In addition, the presence of a self-weight distributed load causes a static deflection of the riser. This introduces quadratic nonlinearity, which in addition to the stretching nonlinearity, can lead to various internal resonance scenarios among the various vibration modes resulting into energy transfer among them. Examples of these internal resonances are 1:1 (one-to-one), 2:1 (two-to-one), and 3:1 (three-to-one) resonances. Internal resonances have been studied in different structures, such as beams [2-6], cables [7-12], and plates [13]. The variable axial load in the riser is often simplified by its average value [14] resulting into a beam equation subjected to a constant axial load.

This research work focuses on the case of two-to-one internal resonance. Related research in beams includes the work of Tien et al. [15] who theoretically examined the two-to-one internal resonance of a shallow arch beam under harmonic excitation using the averaging method. They showed that, using the Menlikov technique, the modulated system exhibits a Hopf bifurcation, which leads to chaotic motion via period doubling bifurcation. Chin et al. [5] studied the two-to-one internal resonance of a hinged-hinged buckled beam under parametric excitation considering the influence of the quadratic nonlinearity only and using the multiple time scales method (MTS). They demonstrated that when the critical forcing amplitude is exceeded the system undergoes Hopf bifurcation and series of period doubling, symmetry breaking, and cyclic folding bifurcations leading to 
chaotic behavior. In addition, they reported that the two-to-one internal resonance cannot be excited in buckled beams for a fixed-fixed configuration. Öi and Pakdemirli [3] studied the two-to-one internal resonance of an arch beam resting on elastic foundation and found that the activation of the internal resonance depends on the type of assumed initial shape. Due to the symmetry between the initial shape and the mode shape, they found that the trigonometric initial solution cannot activate the internal resonance while the parabolic initial shape activates it. More recently, Xiong et al. [16] studied the influence of viscoelasticity on the two-to-one internal resonance of a post buckled beam. In their work, a two-term MTS expansion was applied on the discretized equation to analyze the effect of the viscoelastic damping. The result showed that viscoelasticity diminishes the response of the system and improves its stability. In another work, Zhuangpeng et al. [17] investigated the two-to-one internal resonance of an arch beam with constrained boundaries. The end constrains resulted into breaking the symmetry of the modes and activating the internal resonance. Furthermore, Ma et al. [18] studied the influence of soil-beam interaction of a pile immersed in the soil using the MTS method while considering the two-to-one internal resonance. The nonlinear interaction between the soil and the beam resulted into rich dynamics affecting the dynamic bifurcations of the beam. Recent works in this context include the work of Sahoo et al. [19] who studied the three-to-one internal resonance of a viscoelastic travelling beam under combined parametric excitations. In addition, $\mathrm{Yu}$ et al. [20] investigated the two-to-one internal resonance of an L-shaped beam under primary resonance. They utilized the energy-phase method to characterize the chaotic motion and phase jumps of the response. Furthermore, Zhu et al. [21] examined the three-to-one internal 
resonance of an axially moving sandwiched beam subjected to parametric excitations.

On the subject of a riser structure, where self-weight axial force is considered, Neto and Mazzilli [22] developed an invariant manifold technique along with MTS to establish the nonlinear modes to analyze the riser dynamics under two-to-one internal resonance. Their work accounted for the quadratic nonlinearity where the influence of the internal resonance is present. Results showed good agreement when compared to long time integration. Srinil et al. [2325] investigated the vortex-induced vibration of a catenary riser when the in-plane and out-of-plane frequencies are tuned to two-to-one. The results showed that the influence of the static solution causes the amplitude of the response to be higher when compared to the top tension risers in uniform and shear currents. Other works on this subject include parametric analysis of risers using the MTS method due to the effect of heave excitation [26,27] or internal flow [28].

Due to the lack of extensive research works addressing internal resonances in risers, this research investigates the two-to-one internal resonance of an inclined riser accounting for the variable axial load and mid-plane stretching. The model accounts for the distributed weight that causes a static deflection of the riser. The applied top tension and the riser's configuration are tuned such that the ratio of the third-to-first natural frequencies matches two. In addition, to reduce the computational challenges involved in resolving the fluid forces from a VIV problem, we assume the lift induced forces to be a simple harmonic excitation. Then, the nonlinear equation is treated using three-term expansion in the multiple time scales method to account for the influence of the cubic nonlinearity.

The structure of this paper is as follows: The mathematical formulation of the riser is discussed in Section 2. In Section 3, the perturbation solution of the 
nonlinear equation is addressed. Next, validation of the results by comparing the multiple scales solution to long time integration is shown in Section 4 along with more parametric results and discussions. Section 5 presents concluding remarks.

\section{Mathematical Formulation}

We consider analyzing the interactions in the inclined riser shown in Fig. 1.

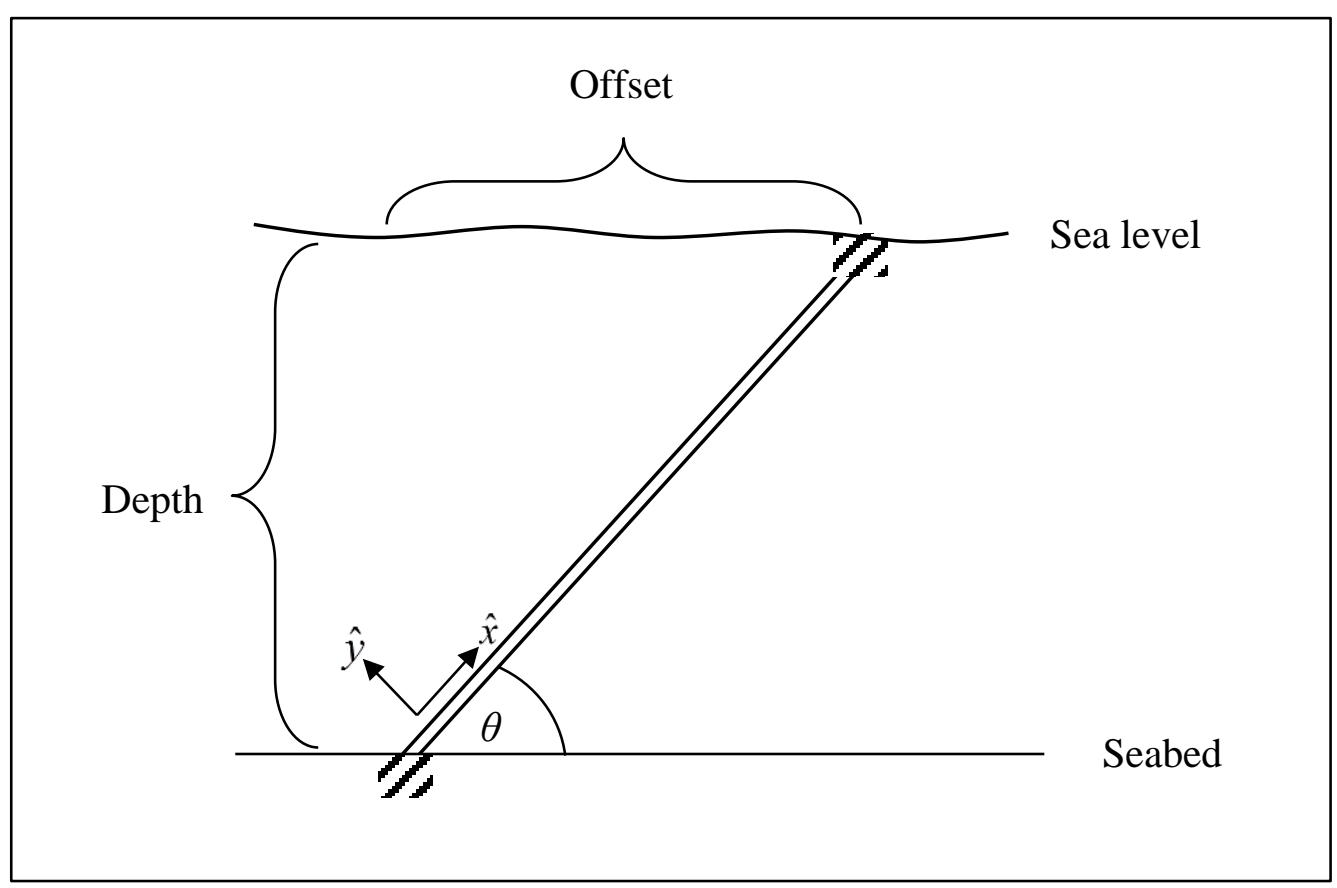

Figure 1. Schematic of the inclined riser.

The riser is modeled as an Euler-Bernoulli beam with variable axial tension and nonlinear mid-plane stretching. Then, the riser equation governing the deflection $\hat{y}$ along the position $\hat{x}$ becomes [29]

$$
\begin{aligned}
& m \frac{\partial^{2} \hat{y}}{\partial \hat{t}^{2}}+E I \frac{\partial^{4} \hat{y}}{\partial \hat{x}^{4}}-\left(\left(T_{e}-W e \operatorname{Sin}(\theta)(L-\hat{x})\right)+\frac{E A_{p}}{2 L} \int_{0}^{L}\left[\left(\frac{\partial \hat{y}}{\partial \hat{x}}\right)^{2}\right] d \hat{x}\right)\left(\frac{\partial^{2} \hat{y}}{\partial \hat{x}^{2}}\right)-\operatorname{WeSin}(\theta)\left(\frac{\partial \hat{y}}{\partial \hat{x}}\right) \\
& +\bar{c} \frac{\partial \hat{y}_{d}}{\partial \hat{t}}=F_{d}-W e \operatorname{Cos}(\theta)
\end{aligned}
$$

Subject to the boundary condition

$$
\begin{gathered}
\frac{\partial \hat{y}}{\partial \hat{x}}=0 \text { at } \hat{x}=0 \text { and } \hat{x}=L \\
\hat{y}(\hat{x}, \hat{t})=0 \text { at } \hat{x}=0 \text { and } \hat{x}=L
\end{gathered}
$$


where $m$ is the total mass per unit length of the riser defined by $m=\rho_{e} A_{e}+\rho_{p} A_{p}$, $\rho_{e}$ is the density of sea water, $A_{e}$ is the area of the pipe based on the outside diameter, $\rho_{p}$ is the pipe material density, $A_{p}$ is the cross section area of the pipe, $\hat{t}$ is the time, $E I$ is the flexural rigidity, $T_{e}$ is the applied tension, We is the riser apparent weight defined by $W e=\left(\rho_{p} A_{p}-\rho_{e} A_{e}\right) g, g$ is the gravitational constant, $\theta$ is the inclination angle, $L$ is the length of the pipe, $\bar{c}$ is the structural damping, and $F_{d}$ is the dynamic applied load. The axial tension of the riser is assumed to vary linearly with the weight of the pipe. We assume that the sea current flow is orthogonal to the plane of curvature of the riser and, thereby, the influence of static drag is not considered in the analysis because it has minimal effect. In addition, the influence of the parametric (heave) excitation is neglected due to the application of a moderate static tension. Also, practically, a heave-compensation system is commonly installed on the riser-platform system to suppress the heave excitation. We assume the total riser deflection $\hat{y}_{t}(\hat{x}, \hat{t})$ solution composed of a static component $\hat{y}_{s}(\hat{x})$ superimposed to a dynamic component $\hat{y}_{d}(\hat{x}, \hat{t})$ as follows

$$
\hat{y}_{t}(\hat{x}, \hat{t})=\hat{y}_{s}(\hat{x})+\hat{y}_{d}(\hat{x}, \hat{t})
$$

The static differential equation of the inclined riser is obtained by dropping the time dependent terms in Eq. (1.1), which results into

$$
E I \frac{d^{4} \hat{y}_{s}}{d \hat{x}^{4}}-\left(\left(T_{e}-\operatorname{WeSin}(\theta)(L-\hat{x})\right)+\frac{E A_{p}}{2 L} \int_{0}^{L}\left[\left(\frac{d \hat{y}_{s}}{d \hat{x}}\right)^{2}\right] d \hat{x}\right)\left(\frac{d^{2} \hat{y}_{s}}{d \hat{x}^{2}}\right)-\operatorname{WeSin}(\theta)\left(\frac{d \hat{y}_{s}}{d \hat{x}}\right)=-\operatorname{WeCos}(\theta)
$$

The static equation is solved using the boundary layer perturbation technique, the method of matched asymptotic expansion, assuming a tension-dominant riser and considering clamped boundary conditions. For a more comprehensive study, hinged boundary conditions can also be considered; however, due to the limitation imposed by the boundary-layer perturbation technique, we do not consider the 
hinged boundaries [30]. The dynamic equation of motion of the inclined riser is obtained after dropping the static terms, Eq. (3). The dynamic equation is written after simplification as

$$
\begin{aligned}
& m \frac{\partial^{2} \hat{y}_{d}}{\partial \hat{t}^{2}}+E I \frac{\partial^{4} \hat{y}_{d}}{\partial \hat{x}^{4}}-\left(T_{e}-\operatorname{WeSin}(\theta)(L-\hat{x})\right)\left(\frac{\partial^{2} \hat{y}_{d}}{\partial \hat{x}^{2}}\right)-\operatorname{WeSin}(\theta)\left(\frac{\partial \hat{y}_{d}}{\partial \hat{x}}\right) \\
& \left.-\left(\frac{E A_{p}}{2 L} \int_{0}^{L}\left[\left(\frac{d \hat{y}_{s}}{d \hat{x}}\right)^{2}\right] d \hat{x}+\int_{0}^{L}\left[\left(\frac{\partial \hat{y}_{d}}{\partial \hat{x}}\right)^{2}\right] d \hat{x}+\int_{0}^{L}\left[2 \frac{d \hat{y}_{s}}{d \hat{x}} \frac{\partial \hat{y}_{d}}{\partial \hat{x}}\right] d \hat{x}\right)\right)\left(\frac{\partial^{2} \hat{y}_{d}}{\partial \hat{x}^{2}}\right) \\
& -\left(\frac{E A_{p}}{2 L}\left(\int_{0}^{L}\left[2 \frac{d \hat{y}_{s}}{d \hat{x}} \frac{\partial \hat{y}_{d}}{\partial \hat{x}}\right] d \hat{x}+\int_{0}^{L}\left[\left(\frac{\partial \hat{y}_{d}}{\partial \hat{x}}\right)^{2}\right] d \hat{x}\right)\right)\left(\frac{d^{2} \hat{y}_{s}}{d \hat{x}^{2}}\right)+\bar{c} \frac{\partial \hat{y}_{d}}{\partial \hat{t}}=\bar{F}_{d}
\end{aligned}
$$

For simplification, the following dimensionless variables are introduced

$$
y_{d}=\frac{\hat{y}}{D} \quad x=\frac{\hat{x}}{L} \quad t=\sqrt{\frac{E I}{m L^{4}}} \hat{t}
$$

where $D$ is the diameter of riser pipe. The "hat" denotes the dimensional variables and the variables without the "hat" are dimensionless. Substituting Eq. (5) into (4) yields

$$
\begin{aligned}
& \frac{\partial^{2} y_{d}}{\partial t^{2}}+\frac{\partial^{4} y_{d}}{\partial x^{4}}-\left(\bar{T}-\bar{\sigma}(1-x)-\bar{\eta}\left(\int_{0}^{1}\left(\frac{d y_{s}}{d x}\right)^{2} d x+\int_{0}^{1}\left(\frac{\partial y_{d}}{\partial x}\right)^{2} d \hat{x}+\int_{0}^{1} 2 \frac{d y_{s}}{d x} \frac{\partial y_{d}}{\partial x} d \hat{x}\right)\right)\left(\frac{\partial^{2} y_{d}}{\partial x^{2}}\right)-\bar{\sigma}\left(\frac{\partial y_{d}}{\partial x}\right) \\
& -\left(\bar{\eta}\left(\int_{0}^{1} 2 \frac{d y_{s}}{d x} \frac{\partial y_{d}}{\partial x} d x+\int_{0}^{1}\left(\frac{\partial y_{d}}{\partial x}\right)^{2} d \hat{x}\right)\right)\left(\frac{d^{2} y_{s}}{d x^{2}}\right)+c \frac{\partial y_{d}}{\partial t}=\bar{F}_{d}
\end{aligned}
$$

where



As noted in Eq. (6), the static deflection of the riser introduces a quadratic nonlinearity in addition to the existing cubic nonlinearity, which arises due to the stretch of the riser structure. The next step is to solve the linear eigenvalue problem in order to obtain the natural frequencies and mode shapes from Eq. (6) by dropping damping, forcing, and nonlinear terms yielding

$$
\frac{\partial^{2} y_{d}}{\partial t^{2}}+\frac{\partial^{4} y_{d}}{\partial x^{4}}+\left(\bar{T}_{s}-\bar{\sigma} x-\bar{\eta} \int_{0}^{1}\left(\frac{d y_{s}}{d x}\right)^{2} d x\right) \frac{\partial^{2} y_{d}}{\partial x^{2}}-\bar{\eta}\left(\int_{0}^{1} 2 \frac{d y_{s}}{d x} \frac{\partial y_{d}}{\partial x} d x\right)\left(\frac{d^{2} y_{s}}{d x^{2}}\right)-\bar{\sigma} \frac{\partial y_{d}}{\partial x}=0
$$


where $\bar{T}_{s}=\bar{\sigma}-\bar{T}$. The mode shapes and the natural frequencies of the structure are extracted by solving Eq. (8), using fifteen beam mode shapes, in a Galerkin method [31].

The riser structure is subjected to vortex-induced vibrations in the cross-flow direction. Several semi-empirical formulas have been developed $[32,33]$ to reduce the level of fluid dynamics computations. However, in this work, these formulations increase the complexity of the analysis that may hinder in-depth study of the nonlinear interaction problems in the riser structure. Therefore, we choose the VIV excitation force to be of the form [1]

$$
\bar{F}_{d}=f \operatorname{Cos}(\Omega t)
$$

where $f=\frac{L^{4} C_{L} \rho_{e} U^{2}}{2 E I}, U$ is the external current velocity, and $C_{L}$ is the average lift coefficient.

\section{Two-To-One Internal Resonance (Perturbations Analysis)}

In this section, we analyze the influence of the quadratic and cubic nonlinearities via the method of multiple scales where the expansion is applied directly on the integral partial differential equation of Eq. (6). Accordingly, we seek a three-order expansion of the form

$$
y_{d}(x, t ; \varepsilon)=\varepsilon y_{1}\left(x, T_{0}, T_{1}, T_{2}\right)+\varepsilon^{2} y_{2}\left(x, T_{0}, T_{1}, T_{2}\right)+\varepsilon^{3} y_{3}\left(x, T_{0}, T_{1}, T_{2}\right)
$$

where $\varepsilon$ is introduced as a bookkeeping parameter, $T_{0}=\varepsilon t, T_{1}=\varepsilon^{2} t$, and $T_{2}=\varepsilon^{3} t$. Due to the static deflection, a large difference is observed between the magnitudes of the quadratic and cubic nonlinearities. Thus, the scale chosen at Eq. (10) determines that the quadratic nonlinearity is at $\varepsilon^{2}$ and the cubic nonlinearity is at 
$\varepsilon^{3}$ and the forcing and damping are ordered as $\varepsilon^{2} f \cos (\Omega t)$ and $\varepsilon c$, respectively. We substitute Eq. (10) into Eq. (6) to obtain

Order $\varepsilon$

$$
\ell\left(y_{1}\right)=D_{0}^{2} y_{1}+2 \sqrt{\beta} v D_{0} y_{1}^{\prime}+y_{1}^{i v}+\bar{T}_{s} y_{1}^{\prime \prime}-x \bar{\sigma} y_{1}^{\prime \prime}-\bar{\sigma} y_{1}^{\prime}-2 \eta y_{s}^{\prime \prime}\left(\int_{0}^{1} y_{s}^{\prime} y_{1}^{\prime} \mathrm{d} x\right)=0
$$

Order $\varepsilon^{2}$

$$
\ell\left(y_{2}\right)=-c D_{0} y_{1}-2 D_{0} D_{1} y_{1}-2 \sqrt{\beta} v D_{1} y_{1}^{\prime}+\eta y_{s}^{\prime \prime}\left(\int_{0}^{1}\left(y_{1}^{\prime}\right)^{2} \mathrm{~d} x\right)+2 \eta y_{1}^{\prime \prime}\left(\int_{0}^{1} y_{s}^{\prime} y_{1}^{\prime} \mathrm{d} x\right)+\frac{f}{2} e^{i \Omega T_{0}}
$$

Order $\varepsilon^{3}$

$$
\begin{aligned}
\ell\left(y_{3}\right)= & -D^{2}{ }_{1} y_{1}-2 D_{0} D_{2} y_{1}-2 D_{0} D_{1} y_{2}-c D_{1} y_{1}-c D_{0} y_{2}+\eta y_{1}^{\prime \prime} \int_{0}^{1}\left(y_{1}^{\prime}\right)^{2} \mathrm{~d} x \\
& +2 \eta\left(y_{2}{ }^{\prime \prime} \int_{0}^{1} y_{s}^{\prime} y_{1}^{\prime} \mathrm{d} x+y_{s}^{\prime \prime} \int_{0}^{1} y_{1}^{\prime} y_{2}^{\prime} \mathrm{d} x+y_{1}^{\prime \prime} \int_{0}^{1} y_{s}^{\prime} y_{2}^{\prime} \mathrm{d} x\right)
\end{aligned}
$$

where the derivatives are defined as $D_{0}^{2}=\frac{\partial}{\partial T_{0}^{2}}, D_{0}=\frac{\partial}{\partial T_{0}}, D_{1}^{2}=\frac{\partial}{\partial T_{1}^{2}}, D_{1}=\frac{\partial}{\partial T_{1}}$ , $D_{2}=\frac{\partial}{\partial T_{2}}$ and ()'denotes the derivative with respect to $x$ and $\ell()$ defines the linear operator given by Eq. (11). It is observed that the first order Eq. (11) is equivalent to the eigenvalue problem defined by Eq. (8). Then, at higher orders, the coupling between the modes will occur due to the activation of the internal resonance. Therefore, the solution to Eq. (11) is written as the superposition of two different modes

$$
\begin{aligned}
y_{1}= & A_{n}\left(T_{1}, T_{2}\right) \phi_{n}(x) e^{i \omega_{n} T_{0}}+A_{m}\left(T_{1}, T_{2}\right) \phi_{m}(x) e^{i \omega_{m} T_{0}} \\
& +\bar{A}_{n}\left(T_{1}, T_{2}\right) \phi_{n}(x) e^{-i \omega_{n} T_{0}}+\bar{A}_{m}\left(T_{1}, T_{2}\right) \phi_{m}(x) e^{-i \omega_{m} T_{0}}
\end{aligned}
$$

where $A_{n}\left(T_{1}, T_{2}\right)$ and $A_{m}\left(T_{1}, T_{2}\right)$ are the amplitudes associated with modes $n$ and $m$, respectively, $\phi_{n}(x)$ and $\phi_{m}(x)$ are the mode shapes, and $\omega_{n}$ and $\omega_{m}$ are the natural frequencies. To determine the solvability condition at the second order, Eq. (14) is substituted into Eq. (12) to obtain 


$$
\begin{aligned}
\ell\left(y_{2}\right) & =-\left(\mathrm{i} \omega_{m} c \phi_{m}(x) A_{m}+2 \mathrm{i} \omega_{m} \phi_{m}(x) D_{1} A_{m}\right) e^{i \omega_{m} T_{0}}-\left(\mathrm{i} \omega_{n} c \phi_{n}(x) A_{n}+2 \mathrm{i} \omega_{n} \phi_{n}(x) D_{1} A_{n}\right) e^{i \omega_{n} T_{0}} \\
& +\left(\eta y_{s}^{\prime \prime}\left(\int_{0}^{1}\left(\phi_{m}^{\prime}\right)^{2} \mathrm{~d} x\right)+2 \eta \phi_{m}^{\prime \prime}\left(\int_{0}^{1} y_{s}^{\prime} \phi_{m}^{\prime} \mathrm{d} x\right)\right) A_{m}{ }^{2} \mathrm{e}^{2 i T_{0} \omega_{m}}+2 \Lambda_{n m} A_{n} \bar{A}_{m} \mathrm{e}^{i T_{0}\left(\omega_{n}-\omega_{m}\right)} \\
& +\frac{f}{2} e^{i \Omega T_{0}}+c c+N S T
\end{aligned}
$$

where $c c$ is the complex conjugate of the expression, NST denotes the non-secular terms, and $\Lambda_{n m}$ is defined as

$$
\Lambda_{n m}=\left(\eta \phi_{m}^{\prime \prime} \int_{1}^{0} y_{s}^{\prime} \phi_{n}^{\prime} \mathrm{d} x+\eta \phi_{n}^{\prime \prime} \int_{1}^{0} y_{s}^{\prime} \phi_{m}^{\prime} \mathrm{d} x+\eta y_{s}^{\prime \prime} \int_{1}^{0} \phi_{m}^{\prime} \phi_{n}^{\prime} \mathrm{d} x\right)
$$

We consider the case when the excitation is around the first primary resonance defined by $\Omega=\omega_{m}+\varepsilon^{2} \alpha$, and the two-to-one internal resonance is given by $\omega_{n}=2 \omega_{m}+\delta \varepsilon^{2}$, where $\alpha$ and $\delta$ are detuning parameters. To obtain the solvability condition, the linear adjoint problem of Eq. (11) needs to be solved for $\phi_{j}(x) e^{ \pm i \omega_{j} T_{0}}$. The proof provided in Appendix A shows that the linear problem of Eq. (11) is self-adjoint. Then, we substitute $\omega_{n}=2 \omega_{m}+\delta \varepsilon$ and $\Omega=\omega_{m}+\varepsilon \alpha$ in Eq. (15) and demand that the right hand side of the equation is orthogonal to $\phi_{m}(x)$ $e^{-i \omega_{m} T_{0}}$ and $\phi_{n}(x) e^{-i \omega_{n} T_{0}}$ while integrating to reduce to

$$
\begin{aligned}
2 \mathrm{i} \omega_{m} D_{1} A_{m} & =-\mathrm{i} \mu_{1} \omega_{m} A_{m}+R_{1} A_{n} \bar{A}_{m} \mathrm{e}^{i \delta T_{1}}+F_{m} e^{i \alpha T_{1}} \\
2 \mathrm{i} \omega_{n} D_{1} A_{n} & =-\mu_{2} \mathrm{i} \omega_{n} A_{n}+R_{2} A_{m}{ }^{2} \mathrm{e}^{-i \delta T_{1}}
\end{aligned}
$$

where the coefficients $R_{i}, \mu_{1}, \mu_{2}, F_{k}$ are defined by

$$
\begin{aligned}
& R_{1}=\int_{0}^{1} \phi_{m} \Lambda_{n m} \mathrm{~d} x, \quad \mu_{1}=\int_{0}^{1} c \phi_{m}^{2} \mathrm{~d} x, \quad \mu_{2}=\int_{0}^{1} c \phi_{n}^{2} \mathrm{~d} x, \quad F_{k}=\frac{1}{2} \int_{0}^{1} f \phi_{k} \mathrm{~d} x, \\
& R_{2}=\int_{0}^{1} \phi_{n}\left(\eta y_{s}^{\prime \prime}\left(\int_{0}^{1} \phi_{m}^{\prime 2} \mathrm{~d} x\right)+2 \eta \phi_{m}^{\prime \prime}\left(\int_{0}^{1} y_{s}^{\prime} \phi_{m}^{\prime} \mathrm{d} x\right)\right) \mathrm{d} x
\end{aligned}
$$

From Eq. (17) and Eq. (18), the necessary condition to activate the two-to-one internal resonance is given by non-zero coupling coefficients $R_{1}$ and $R_{2}$. In the 
absence of higher order nonlinearity, one can stop the analysis up to this order of $\varepsilon^{2}$ and analyze the modulation equations, Eq. (17) and Eq. (18). However, the mid-plane stretching has a cubic nonlinearity to be accounted for. For that reason, we extend the analysis to $\varepsilon^{3}$ and consider the particular solution of the nonsecular terms in Eq. (15). The particular solution is written as

$$
\begin{aligned}
y_{2}= & \psi_{1}(x) A_{m} A_{n} \mathrm{e}^{i\left(\omega_{n}+\omega_{m}\right) T_{0}}+\psi_{2}(x) A_{n}{ }^{2} \mathrm{e}^{i 2 \omega_{n} T_{0}}+\psi_{3}(x) A_{m} \bar{A}_{m}+\psi_{4}(x) A_{n} \bar{A}_{n} \\
& +B_{m} \phi_{m}(x) \mathrm{e}^{\mathrm{i} T_{0} \omega_{m}}+B_{n} \phi_{n}(x) \mathrm{e}^{\mathrm{i} T_{0} \omega_{n}}+c c
\end{aligned}
$$

where $\psi_{1}, \psi_{2}, \psi_{3}, \psi_{4}$ are given by solving the following boundary value problems:

$$
\begin{aligned}
& \psi_{1}(x): \mathrm{H}\left[\omega_{n}+\omega_{m}\right]=2 \Lambda_{n m} \\
& \psi_{2}(x): \mathrm{H}\left[2 \omega_{n}\right]=\Lambda_{n} \\
& \psi_{3}(x): \mathrm{H}[0]=\Lambda_{m} \\
& \psi_{4}(x): \mathrm{H}[0]=\Lambda_{n}
\end{aligned}
$$

and $\mathrm{H}$ is defined as

$$
\mathrm{H}\left[\omega_{i}\right]=H^{i v}-N H^{\prime \prime}-2 \alpha_{1} w_{s}^{\prime \prime}\left(\int_{0}^{1} w_{s}^{\prime} H^{\prime} \mathrm{d} x\right)-\omega_{i}^{2} H
$$

where $\Lambda_{i}$ is defined as

$$
\Lambda_{i}=\eta y_{s}^{\prime \prime}(x)\left(\int_{0}^{1} \phi_{i}^{\prime}(x)^{2} \mathrm{~d} x\right)+2 \eta \phi_{i}^{\prime \prime}(x)\left(\int_{0}^{1} \phi_{i}^{\prime}(x) y_{s}^{\prime}(x) \mathrm{d} x\right)
$$

The boundary value problems in Eq. (21.1) - Eq. (21.4) are subjected to clampedclamped boundary conditions defined by

$$
\psi_{i}(0)=0, \psi_{i}(1)=0, \frac{d \psi_{i}(0)}{d x}=0, \frac{d \psi_{i}(1)}{d x}=0
$$

In addition, these equations are solved using Galerkin procedure prescribed in [31]. The expansion of the multiple scales solution up to $\varepsilon^{3}$, in the two-to one internal resonance, yields secular terms that are not derivable from the time averaged Lagrangian. Therefore, $B_{m}\left(T_{1}, T_{2}\right), B_{n}\left(T_{1}, T_{2}\right)$ are introduced as a correction to satisfy the Lagrangian formulation at the third order [34]. 
Substituting the solution from Eq. (14) and Eq. (20) into the third order Eq. (13) while considering $\omega_{n}=2 \omega_{m}+\varepsilon \delta, \Omega=\omega_{m}+\varepsilon \alpha$ and multiplying with the adjoint $\phi_{m}(x) e^{-i \omega_{m} T_{0}}$ and $\phi_{n}(x) e^{-i \omega_{n} T_{0}}$ yield the solvability condition at the third order given by

$$
\begin{aligned}
2 \mathrm{i} \omega_{m} D_{2} A_{m}= & -\mu_{1} D_{1} A_{m}-D_{1}^{2} A_{m}+K_{1} A_{m} A_{n} \bar{A}_{n}+K_{2} A_{m}{ }^{2} \bar{A}_{m}-\mathrm{i} \mu_{1} \omega_{m} B_{m}-2 \mathrm{i} \omega_{m} D_{1} B_{m} \\
& +R_{1} \mathrm{e}^{\mathrm{i} \delta T_{1}} B_{n} \bar{A}_{m}+R_{1} \mathrm{e}^{\mathrm{i} \delta T_{1}} A_{n} \bar{B}_{m} \\
2 \mathrm{i} \omega_{n} D_{2} A_{n}= & -\mu_{1} D_{1} A_{n}-D_{2}^{2} A_{n}+K_{3} A_{m} A_{n} \bar{A}_{m}+K_{4} A_{n}{ }^{2} \bar{A}_{n}+2 R_{2} \mathrm{e}^{-\mathrm{i} \delta T_{1}} A_{m} B_{m}-\mathrm{i} \mu_{1} \omega_{n} B_{n} \\
& -2 \mathrm{i} \omega_{n} D_{1} B_{n}
\end{aligned}
$$

where the coefficients $K_{1}, K_{2}, K_{3}$ and $K_{4}$ are defined in Appendix B. To ensure that the modulation equation satisfies the Lagrangian, we demand

$$
\begin{aligned}
& D_{1}\left[2 \mathrm{i} \omega_{m} B_{m}\left(T_{1}, T_{2}\right)+D_{1} A_{m}\left(T_{1}, T_{2}\right)\right]=0 \\
& D_{1}\left[2 \mathrm{i} \omega_{n} B_{n}\left(T_{1}, T_{2}\right)+D_{1} A_{n}\left(T_{1}, T_{2}\right)\right]=0
\end{aligned}
$$

Equations (25.1) and (25.2) yield

$$
\begin{aligned}
& 2 \mathrm{i} \omega_{m} B_{m}\left(T_{1}, T_{2}\right)+D_{1} A_{m}\left(T_{1}, T_{2}\right)=c_{1}\left(T_{2}\right) \\
& 2 \mathrm{i} \omega_{n} B_{n}\left(T_{1}, T_{2}\right)+D_{1} A_{n}\left(T_{1}, T_{2}\right)=c_{2}\left(T_{2}\right)
\end{aligned}
$$

From Eq. (17) and Eq. (18), we note that $D_{1} A_{m}\left(T_{1}, T_{2}\right)$ and $D_{1} A_{n}\left(T_{1}, T_{2}\right)$ have no explicit dependence on $T_{2}$. Then, $c_{1}\left(T_{2}\right)=c_{2}\left(T_{2}\right)=0$, which yields

$$
\begin{aligned}
& B_{m}=-\frac{\mathrm{i} \mu_{1}}{4 \omega_{m}} A_{m}+\frac{R_{1}}{4 \omega_{m}^{2}} A_{n} \bar{A}_{m} \mathrm{e}^{\mathrm{i} \delta T_{1}}+\frac{F_{m}}{4 \omega_{m}^{2}} \mathrm{e}^{\mathrm{i} \alpha T_{1}} \\
& B_{n}=-\frac{\mathrm{i} \mu_{1}}{4 \omega_{n}} A_{n}+\frac{R_{2}}{4 \omega_{n}^{2}} A_{m}{ }^{2} \mathrm{e}^{-\mathrm{i} \delta T_{1}}
\end{aligned}
$$

The next step is to combine the solutions from Eq. (17), Eq. (18), Eq. (23), Eq. (24), Eq. (27.1), and Eq. (27.2) using the method of reconstitution [35] defined by

$$
\frac{d A_{i}}{d t}(t, \varepsilon)=\varepsilon D_{1} A_{i}+\varepsilon^{2} D_{2} A_{i}
$$


Setting $\varepsilon$ to unity and after further manipulation, the modulation equations are given by

$$
\begin{aligned}
\frac{d A_{m}}{d t}= & -\left(\frac{\mu_{1}}{2}+\frac{\mathrm{i} \mu_{1}^{2}}{8 \omega_{m}}\right) A_{m}-\left(\frac{\mathrm{i} K_{2}}{2 \omega_{m}}+\frac{\mathrm{i} R_{1} R_{2}}{8 \omega_{m} \omega_{n}^{2}}\right) A_{m}^{2} \bar{A}_{m}-\left(\frac{\mathrm{i} K_{1}}{2 \omega_{m}}+\frac{\mathrm{i} R_{1}^{2}}{8 \omega_{m}^{3}}\right) A_{m} A_{n} \bar{A}_{n} \\
& +\left(\frac{\mu_{1} R_{1}}{4 \omega_{m}^{2}}-\frac{\mu_{1} R_{1}}{8 \omega_{m} \omega_{n}}-\frac{\mathrm{i} R_{1}}{2 \omega_{m}}\right) A_{n} \bar{A}_{m} \mathrm{e}^{\mathrm{i} \delta T_{1}}-\frac{\mathrm{i} R_{1} F_{m}}{8 \omega_{m}^{3}} A_{n} \mathrm{e}^{\mathrm{i}(\alpha-\delta) T_{1}}+\left(\frac{\mu_{1}}{8 \omega_{m}^{2}}-\frac{\mathrm{i}}{2 \omega_{m}}\right) \mathrm{e}^{\mathrm{i} \alpha T_{1}} F_{m} \\
\frac{d A_{n}}{d t}= & -\left(\frac{\mu_{1}}{2}+\frac{\mathrm{i} \mu_{1}^{2}}{8 \omega_{n}}\right) A_{n}-\left(\frac{\mathrm{i} K_{3}}{2 \omega_{n}}+\frac{\mathrm{i} R_{1} R_{2}}{4 \omega_{m}^{2} \omega_{n}}\right) A_{m} A_{n} \bar{A}_{m}-\frac{\mathrm{i} K_{4}}{2 \omega_{n}} A_{n}^{2} \bar{A}_{n}-\frac{\mathrm{i} R_{2} F_{m}}{4 \omega_{m}^{2} \omega_{n}} A_{m} \mathrm{e}^{\mathrm{i} T_{1}(\alpha-\delta)} \\
& +\left(\frac{\mu_{1} R_{2}}{8 \omega_{n}^{2}}-\frac{2 \mu_{1} R_{2}}{4 \omega_{m} \omega_{n}}-\frac{2 \mathrm{i} R_{2}}{2 \omega_{n}}\right) A_{m}^{2} \mathrm{e}^{-\mathrm{i} \delta T_{1}}
\end{aligned}
$$

Equation (29.1) and (29.2) resemble the solvability condition that captures the influence of the second and third orders respectively. Then, we substitute the polar transformation given by

$$
\begin{aligned}
& A_{m}=\frac{1}{2} a_{m} e^{i\left(\beta_{m}+\alpha t\right)} \\
& A_{n}=\frac{1}{2} a_{n} e^{i\left(\beta_{n}+(2 \alpha-\delta) t\right)}
\end{aligned}
$$

Substituting Eq. (30.1) and Eq. (30.2) into Eq. (29.1) and Eq. (29.2) yields

$$
\begin{aligned}
a_{m}^{\prime}= & -\frac{\mu_{1}}{2} a_{m}-\frac{R_{1} F_{m}}{8 \omega_{m}^{3}} a_{n} \sin \left(\beta_{m}-\beta_{n}\right)+\frac{\mu_{1} F_{m}}{4 \omega_{m}^{2}} \cos \left(\beta_{m}\right)-\frac{F_{m}}{\omega_{m}} \sin \left(\beta_{m}\right) \\
& -\frac{R_{1}}{4 \omega_{m}} a_{m} a_{n} \sin \left(2 \beta_{m}-\beta_{n}\right)+\left(\frac{\mu_{1}}{8 \omega_{m}^{2}}-\frac{\mu_{1}}{16 \omega_{m} \omega_{n}}\right) R_{1} a_{m} a_{n} \cos \left(2 \beta_{m}-\beta_{n}\right) \\
a_{m} \beta_{m}^{\prime}= & -\left(\alpha+\frac{\mu_{1}^{2}}{8 \omega_{m}}\right) a_{m}-\frac{\mu_{1} F_{m}}{4 \omega_{m}^{2}} \sin \left(\beta_{m}\right)-\frac{F_{m}}{\omega_{m}} \cos \left(\beta_{m}\right)-\left(\frac{R_{1} R_{2}}{32 \omega_{m} \omega_{n}^{2}}+\frac{K_{2}}{8 \omega_{m}}\right) a_{m}^{3} \\
- & \left(\frac{R_{1}^{2}}{32 \omega_{m}^{3}}+\frac{K_{1}}{8 \omega_{m}}\right) a_{m} a_{n}^{2}-\frac{R_{1} F_{m}}{8 \omega_{m}^{3}} a_{n} \cos \left(\beta_{m}-\beta_{n}\right) \\
+ & \left(\frac{\mu_{1} R_{1}}{16 \omega_{m} \omega_{n}}-\frac{\mu_{1} R_{1}}{8 \omega_{m}^{2}}\right) a_{m} a_{n} \sin \left(2 \beta_{m}-\beta_{n}\right)-\frac{R_{1}}{4 \omega_{m}} a_{m} a_{n} \cos \left(2 \beta_{m}-\beta_{n}\right) \\
a_{n}^{\prime}= & -\frac{\mu_{1}}{2} a_{n}+\frac{R_{2} F_{m}}{4 \omega_{m}^{2} \omega_{n}} a_{m} \sin \left(\beta_{m}-\beta_{n}\right)+\left(\frac{R_{2} \mu_{1}}{16 \omega_{n}^{2}}-\frac{R_{2} \mu_{1}}{8 \omega_{m} \omega_{n}}\right) a_{m}^{2} \cos \left(2 \beta_{m}-\beta_{n}\right) \\
& +\frac{R_{2}}{4 \omega_{n}} a_{m}^{2} \sin \left(2 \beta_{m}-\beta_{n}\right)
\end{aligned}
$$




$$
\begin{aligned}
a_{n} \beta_{n}^{\prime}= & \left(\delta-2 \alpha-\frac{\mu_{1}^{2}}{8 \omega_{n}}\right) a_{n}-\frac{R_{2} F_{m}}{4 \omega_{m}^{2} \omega_{n}} a_{m} \cos \left(\beta_{m}-\beta_{n}\right)-\left(\frac{K_{3}}{8 \omega_{n}}+\frac{R_{1} R_{2}}{16 \omega_{m}^{2} \omega_{n}}\right) a_{m}^{2} a_{n}-\frac{K_{4}}{8 \omega_{n}} a_{n}^{3} \\
& -\frac{R_{2}}{4 \omega_{n}} a_{m}^{2} \cos \left(2 \beta_{m}-\beta_{n}\right)+\left(\frac{R_{2} \mu_{1}}{16 \omega_{n}^{2}}-\frac{R_{2} \mu_{1}}{8 \omega_{m} \omega_{n}}\right) a_{m}^{2} \sin \left(2 \beta_{m}-\beta_{n}\right)
\end{aligned}
$$

Equations (31) represent a system of equations that govern the phase and amplitude modulation. The fixed points (equilibrium solutions) of the system are obtained by setting the right-hand side of the equations to zero reducing them to a set of algebraic equations. The equations are solved using a pseudo-arc length continuation method [36]. The system response is then expressed as

$$
\begin{aligned}
y & =y_{s}(x)+a_{m} \phi_{m}(x) \cos \left(\left(\omega_{m}+\alpha\right) t+\beta_{m}\right)+\frac{\mu_{1}}{4 \omega_{m}} a_{m} \phi_{m}(x) \sin \left(\left(\omega_{m}+\alpha\right) t+\beta_{m}\right) \\
& +a_{n} \phi_{n}(x) \cos \left(\left(\omega_{n}+2 \alpha-\delta\right) t+\beta_{n}\right)+\frac{\mu_{2}}{4 \omega_{n}} a_{n} \phi_{n}(x) \sin \left(\left(\omega_{n}+2 \alpha-\delta\right) t+\beta_{n}\right) \\
& +\frac{1}{4} a_{m}^{2} \psi_{3}(x)+\frac{1}{4} a_{n}^{2} \psi_{4}(x)+\frac{1}{2} a_{m} a_{n} \psi_{1}(x) \cos \left(\left(\omega_{m}+\omega_{n}+3 \alpha-\delta\right) t+\beta_{n}+\beta_{m}\right) \\
& +\frac{1}{2} a_{n}^{2} \psi_{2}(x) \cos \left(2\left(\omega_{n}+2 \alpha-\delta\right) t+2 \beta_{n}\right)+\frac{R_{1}}{8 \omega_{m}^{2}} a_{m} a_{n} \phi_{m}(x) \cos \left(\left(\omega_{m}+\alpha\right) t+\beta_{n}-\beta_{m}\right) \\
& +\frac{F_{m}}{2 \omega_{m}^{2}} \phi_{m}(x) \cos \left(\left(\omega_{m}+\alpha\right) t\right)+\frac{R_{2}}{8 \omega_{n}^{2}} a_{m}^{2} \phi_{n}(x) \cos \left(\left(\omega_{n}+2 \alpha-\delta\right) t+2 \beta_{m}\right)
\end{aligned}
$$

To analyze the stability of the system, we express the amplitudes using the complex Cartesian format to avoid numerical issues when the trivial solution is present [13]. This is done by letting

$$
\begin{aligned}
& A_{m}=\frac{1}{2}\left(p_{m}-\mathrm{i} q_{m}\right) \mathrm{e}^{\mathrm{i} \lambda_{1} t} \\
& A_{n}=\frac{1}{2}\left(p_{n}-\mathrm{i} q_{n}\right) \mathrm{e}^{\mathrm{i} \lambda_{2} t}
\end{aligned}
$$

Substituting Eq. (33.1) and Eq. (33.2) into Eq. (29) yields

$$
\begin{aligned}
p_{m}^{\prime} & =-\frac{\mu_{1}}{2} p_{m}-\left(\alpha+\frac{\mu_{1}^{2}}{8 \omega_{m}}\right) q_{m}+\frac{\mu_{1} F_{m}}{4 \omega_{m}^{2}}-\frac{R_{1} F_{m}}{8 \omega_{m}^{3}} q_{n}+\frac{R_{1}}{4 \omega_{m}}\left(q_{m} p_{n}-p_{m} q_{n}\right) \\
& +\left(\frac{\mu_{1} R_{1}}{8 \omega_{m}^{2}}-\frac{\mu_{1} R_{1}}{16 \omega_{m} \omega_{n}}\right)\left(p_{m} p_{n}+q_{m} q_{n}\right)-q_{m} p_{n}^{2}\left(\frac{K_{1}}{8 \omega_{m}}+\frac{R_{1}^{2}}{32 \omega_{m}^{3}}\right)-p_{m}^{2} q_{m}\left(\frac{K_{2}}{8 \omega_{m}}+\frac{R_{1} R_{2}}{32 \omega_{m} \omega_{n}^{2}}\right) \\
& -q_{m}^{3}\left(\frac{K_{2}}{8 \omega_{m}}+\frac{R_{1} R_{2}}{32 \omega_{m} \omega_{n}^{2}}\right)-q_{m} q_{n}^{2}\left(\frac{K_{1}}{8 \omega_{m}}+\frac{R_{1}^{2}}{32 \omega_{m}^{3}}\right)
\end{aligned}
$$




$$
\begin{aligned}
q_{m}^{\prime}= & -\frac{\mu_{1}}{2} q_{m}+\left(\frac{\mu_{1}^{2}}{8 \omega_{m}}+\alpha\right) p_{m}+\frac{R_{1} F_{m}}{8 \omega_{m}^{3}} p_{n}+\frac{F_{m}}{\omega_{m}}-\left(\frac{\mu_{1} R_{1}}{16 \omega_{m} \omega_{n}}-\frac{\mu_{1} R_{1}}{8 \omega_{m}^{2}}\right)\left(p_{m} q_{n}+q_{m} p_{n}\right) \\
& +\frac{R_{1}}{4 \omega_{m}}\left(q_{m} q_{n}+p_{m} p_{n}\right)+p_{m} q_{n}^{2}\left(\frac{K_{1}}{8 \omega_{m}}+\frac{R_{1}^{2}}{32 \omega_{m}^{3}}\right)+p_{m} q_{m}^{2}\left(\frac{K_{2}}{8 \omega_{m}}+\frac{R_{1} R_{2}}{32 \omega_{m} \omega_{n}^{2}}\right) \\
& +p_{m}^{3}\left(\frac{K_{2}}{8 \omega_{m}}+\frac{R_{1} R_{2}}{32 \omega_{m} \omega_{n}^{2}}\right)+p_{m} p_{n}^{2}\left(\frac{K_{1}}{8 \omega_{m}}+\frac{R_{1}^{2}}{32 \omega_{m}^{3}}\right) \\
p_{n}^{\prime}= & -\frac{\mu_{1}}{2} p_{n}-\left((2 \alpha-\delta)+\frac{\mu_{1}^{2}}{8 \omega_{n}}\right) q_{n}-\frac{R_{2} F_{m}}{4 \omega_{m}^{2} \omega_{n}} q_{m}-\frac{R_{2}}{2 \omega_{n}} p_{m} q_{m}+\left(\frac{\mu_{1} R_{2}}{16 \omega_{n}^{2}}-\frac{\mu_{1} R_{2}}{8 \omega_{m} \omega_{n}}\right)\left(p_{m}^{2}-q_{m}^{2}\right) \\
& -p_{m}^{2} q_{n}\left(\frac{K_{3}}{8 \omega_{n}}+\frac{R_{1} R_{2}}{16 \omega_{m}^{2} \omega_{n}}\right)-q_{m}^{2} q_{n}\left(\frac{K_{3}}{8 \omega_{n}}+\frac{R_{1} R_{2}}{16 \omega_{m}^{2} \omega_{n}}\right)-\frac{K_{4}}{8 \omega_{n}} p_{n}^{2} q_{n}-\frac{K_{4}}{8 \omega_{n}} q_{n}^{3} \\
q_{n}^{\prime}= & -\frac{\mu_{1}}{2} q_{n}+\left((2 \alpha-\delta)+\frac{\mu_{1}^{2}}{4 \omega_{n}}\right) p_{n}+\frac{R_{2} F_{m}}{4 \omega_{m}^{2} \omega_{n}} p_{m}+\frac{R_{2}}{4 \omega_{n}}\left(p_{m}^{2}-q_{m}^{2}\right)-p_{m} q_{m}\left(\frac{\mu_{1} R_{2}}{4 \omega_{m} \omega_{n}}-\frac{\mu_{1} R_{2}}{8 \omega_{n}^{2}}\right) \\
+ & p_{m}^{2} p_{n}\left(\frac{K_{3}}{8 \omega_{n}}+\frac{R_{1} R_{2}}{16 \omega_{m}^{2} \omega_{n}}\right)+\frac{K_{4}}{8 \omega_{n}} p_{n} q_{n}^{2}+\frac{K_{3}}{8 \omega_{n}} q_{m}^{2} p_{n}+\frac{K_{4}}{8 \omega_{n}} p_{n}^{3}+\frac{R_{1} R_{2}}{16 \omega_{m}^{2} \omega_{n}} q_{m}^{2} p_{n}
\end{aligned}
$$

where $\lambda_{1}=\alpha$ and $\lambda_{2}=2 \alpha-\delta$. Then, the stability of the system is obtained by examining the eigenvalues of the Jacobian of the system of Eqs. (34) after substituting the fixed points. To further investigate the stability of the response near Hopf bifurcations, the method of normal forms via second order multiple scales (MTS) is applied on Eq. (34.1) - Eq. (34.4) using the procedure prescribed in [37] to reduce to

$$
\begin{aligned}
& r^{\prime}=\kappa_{1}\left(\alpha-\alpha_{c}\right) r+\kappa_{2} r^{3} \\
& \vartheta^{\prime}=\kappa_{3}\left(\alpha-\alpha_{c}\right) r+\kappa_{4} r^{2}
\end{aligned}
$$

where $r$ represents the amplitude of the limit cycle, $\alpha_{c}$ is the value at which Hopf bifurcation occurs, and $\vartheta$ is the phase of the limit cycle. The limit cycle is stable when $\kappa_{2}$ is negative (supercritical Hopf bifurcation), and it is unstable when $\kappa_{2}$ is positive (subcritical Hopf bifurcation). 


\section{Results and Discussion}

\subsection{Natural Frequency Results}

The solution from the eigenvalue problem from Eq. (8) is solved as a first step by varying the inclination angle $\theta$ and the applied tension. The parameters are tuned such that the ratio between the frequencies becomes 2 . The data in Table 1 are used to interpret the results. The linear frequency results are depicted in Figure 2.

Table 1. Values of the geometric and material parameters used in the calculations.

\begin{tabular}{ll}
\hline Parameter & Value \\
\hline Outside Diameter, $\boldsymbol{D}$ & $0.26 \mathrm{~m}$ \\
Inside Diameter, $\boldsymbol{D}_{\boldsymbol{i}}$ & $0.2 \mathrm{~m}$ \\
Modulus of Elasticity, $\boldsymbol{E}$ & $207 \mathrm{GPa}$ \\
Density of Riser, $\boldsymbol{\rho}_{\boldsymbol{p}}$ & $7850 \mathrm{Kg} / \mathrm{m}^{3}$ \\
Density of Sea Water, $\boldsymbol{\rho}_{\boldsymbol{e}}$ & $1025 \mathrm{Kg} / \mathrm{m}^{3}$ \\
Depth of Sea & $150 \mathrm{~m}$ \\
\hline
\end{tabular}

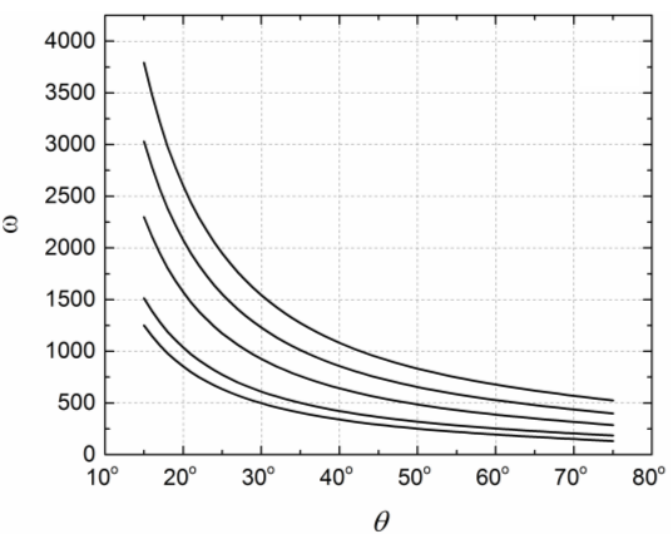

(a)

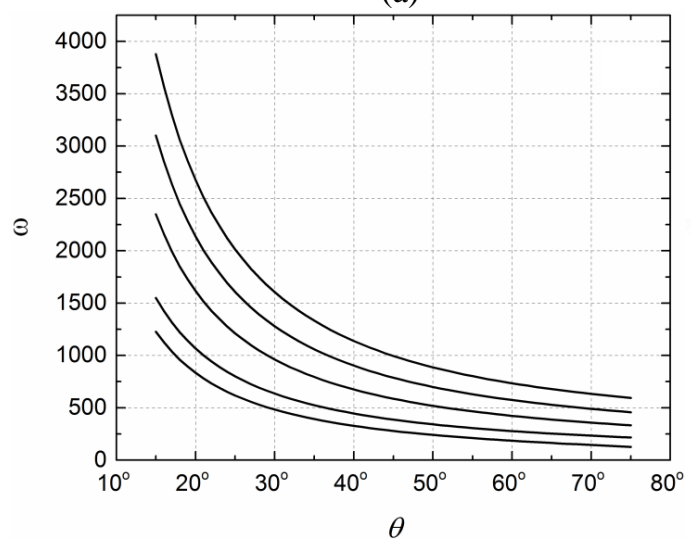

(c)

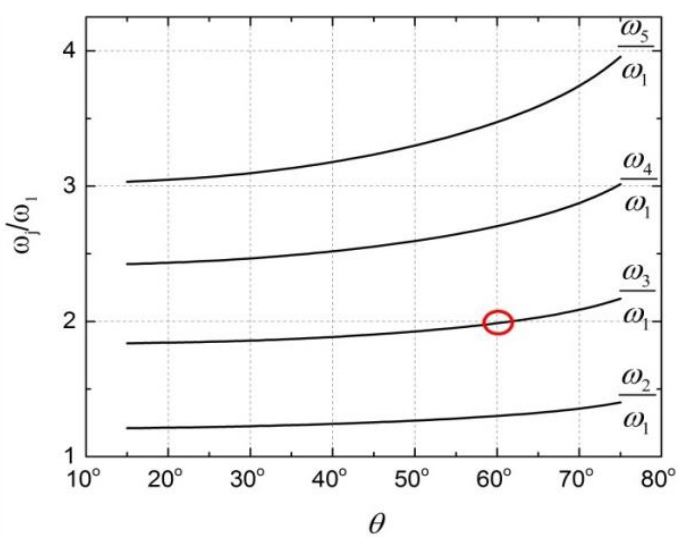

(b)

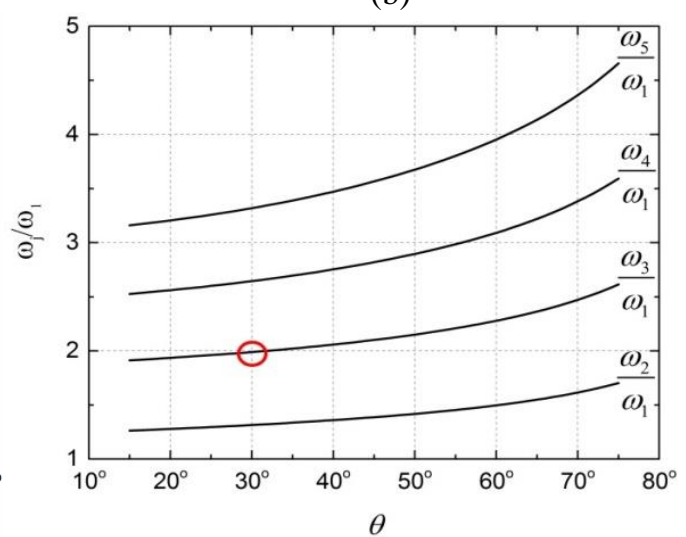

(d) 
Figure 2. Natural frequency values at different inclination angles. (a) Lowest five natural frequencies at $\bar{T}=2 \bar{\sigma}$. (b) Normalized natural frequency values, with respect to the $1^{\text {st }}$ natural frequency, at $\bar{T}=2 \bar{\sigma}$. (c) Lowest five natural frequencies at $\bar{T}=5 \bar{\sigma}$. (d) Normalized natural frequency at $\bar{T}=5 \bar{\sigma}$.

When the inclination angle is changed from lower values (close to horizontal configuration) to the higher angle values (close to vertical configuration), we notice that the frequency values tend to decrease due to the decrease in length and thereby the static deflection of the riser. This is apparent from Fig. 2a and Fig. 2c. Then, examining the frequency ratios with respect to the first natural frequency reveals the possibility of a two-to-one internal resonance between the first and third frequencies (in circle) at $\theta=32^{\circ}$ and $\theta=62^{\circ}$ for tension cases $\bar{T}=2 \bar{\sigma}$ and $\bar{T}=5 \bar{\sigma}$ at Fig. $2 \mathrm{~b}$ and Fig. $2 \mathrm{~d}$, respectively. Since these results are of interest to this study, the configuration angles are fixed at these values and we sweep the applied tension to analyze the nonlinear interactions. The values of the frequencies are presented in Table 2.

Table 2. Frequency values for the two configurations under study.

\begin{tabular}{lllll}
\hline $\bar{T}$ & $\boldsymbol{\theta}$ & $\omega_{1}$ & $\omega_{3}$ & $\delta$ \\
\hline $2 \bar{\sigma}=414.04$ & $62^{\circ}$ & 186.11 & 372.63 & 0.41 \\
\hline $5 \bar{\sigma}=2873.67$ & $32^{\circ}$ & 443.59 & 887.79 & 0.60 \\
\hline
\end{tabular}

In addition, we show the corresponding static and mode shapes plots in Fig. 3 and

Fig. 4. Based on the static deflection in Fig. 3, the maximum deflection of the inclined riser is observed at $x \approx 0.494$ for $\theta=32^{\circ}$ and at $x \approx 0.490$ for $\theta=62^{\circ}$, which is close to the midpoint position of the riser. These results suggest that the influence of the self-weight is reduced at these values of applied tension. 


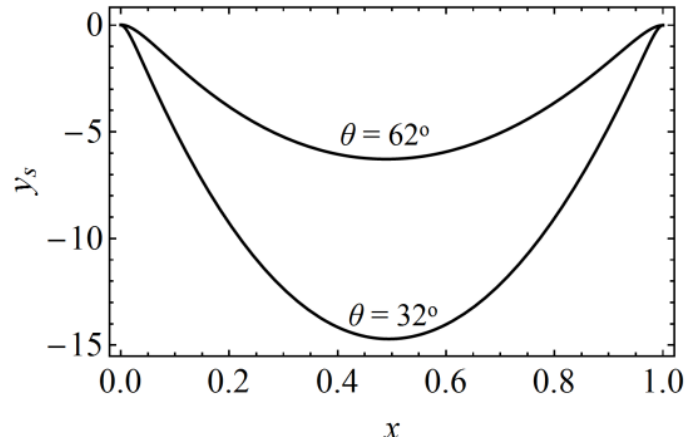

(a)

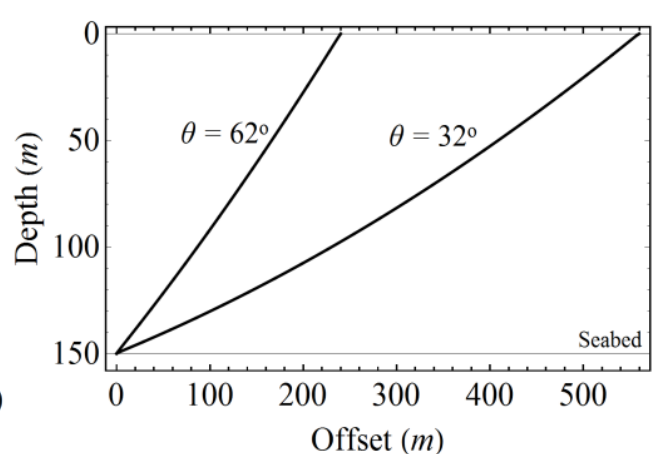

(b)

Figure 3. (a) Static deflection profile along the position of the inclined riser for the configurations in Table 2. (b) Corresponding dimensional static deflection profiles with respect to the depth and offset parameters. The data in Table 1 are utilized to obtain the results.

The results in Fig. 4 show the lowest five mode shapes for $\theta=62^{\circ}$ configuration confirming the fact that the maximum deflection is near the midpoint of the riser. Because the mode shape exhibits similarities to those observed in beams, the dynamic solutions from multiple scales and other method are calculated at the maximum deflection point, i.e. $x \approx 0.494$ for $\theta=32^{\circ}$ and at $x \approx 0.490$ for $\theta=62^{\circ}$.

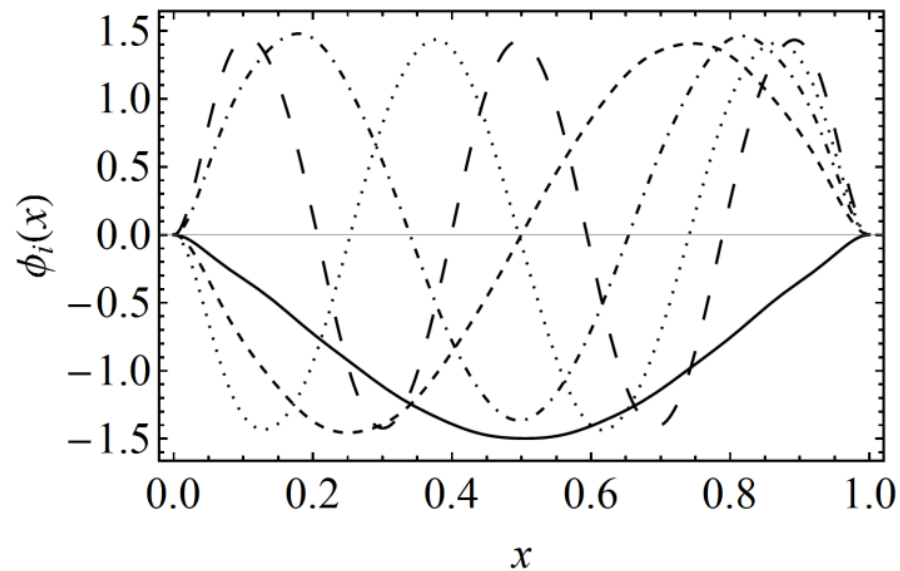

Figure 4. The lowest five mode shapes of the inclined riser at a configuration angle of $\theta=62^{\circ}$. $(-) 1^{\text {st }}$ mode shape, $(\ldots \ldots) 2^{\text {nd }}$ mode shape, $(\ldots \ldots) 3^{\text {rd }}$ mode shape, $(\ldots \ldots.) 4^{\text {th }}$ mode shape, $(-\quad) 5^{\text {th }}$ mode shape.

\subsection{Two-To-One Internal Resonance (Perturbation Results)}

To ensure the validity of the multiple scale solution obtained by Eq. (31), the Galerkin method is applied to discretize Eq. (6), using five mode shapes from Eq. (8), into a set of ordinary differential equations in time, which are then integrated using fourth order Runge-Kutta technique in Matehmatica [38] while updating initial conditions. The result of this comparison is shown in Fig. 5. 


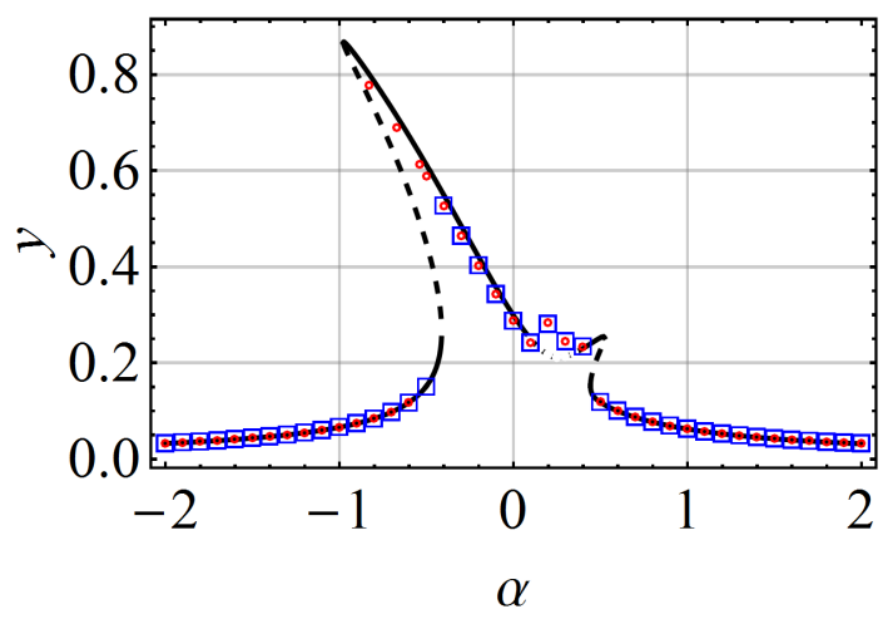

Figure 5. Frequency response curves showing the deflection $y$ as a function of the detuning parameter $\alpha$ for $\theta=62^{\circ}, \mu_{1}=0.08, \mu_{2}=0.08, F_{m}=8$. (-) Stable fixed points, $(--)$ unstable fixed points from the perturbation method. ( $\square$ ) Solutions by long time integration (forward sweep). ( ० ) Solutions by long time integration (backward sweep).

A good agreement is observed between the multiple scales solution and the long time integration results. The multiple scales solution captures the main features of the interaction, i.e., the saddle node jumps at the hardening and the softening bands as well as the unstable bifurcated band. It is noteworthy that a computational challenge was experienced when capturing the solution at the upper softening branch by the Galerkin technique in the backward sweep. This may suggest that the basins of attraction of these solutions are very weak causing the solution to jump to the lower branch. Also, the estimated external current velocity corresponding to the forcing amplitude is $U_{e}=0.09295 \mathrm{~m} / \mathrm{s}$, which suggests that the interaction between the modes can occur at very low velocities.

A further investigation is carried out by plotting the amplitudes corresponding to each mode in each configuration case. These results represent the fixed points obtained by solving the algebraic system of Eqs. (31). The results are shown in Fig. 6. 


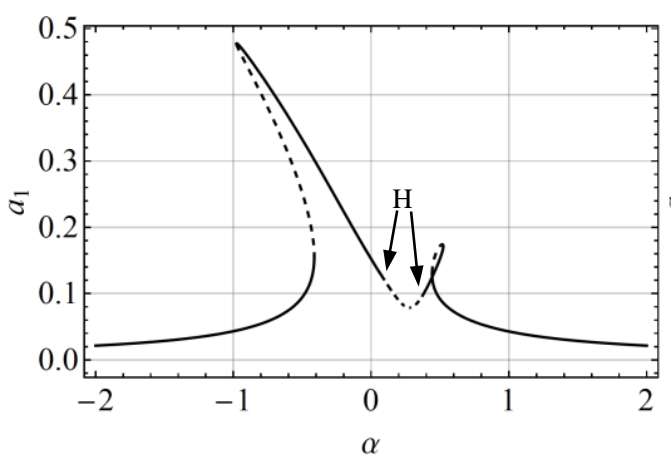

(a)

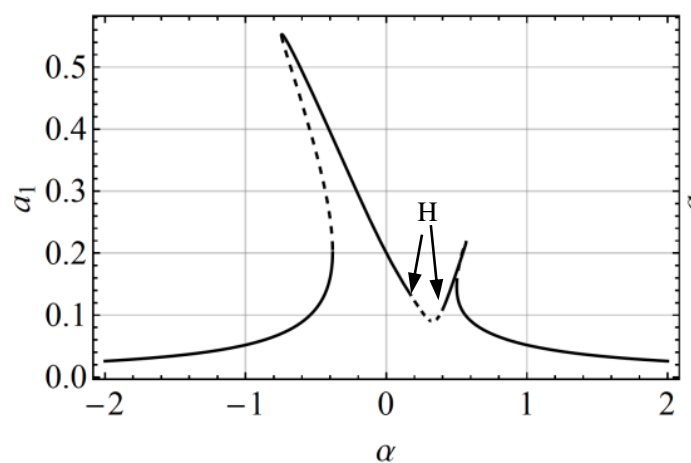

(c)

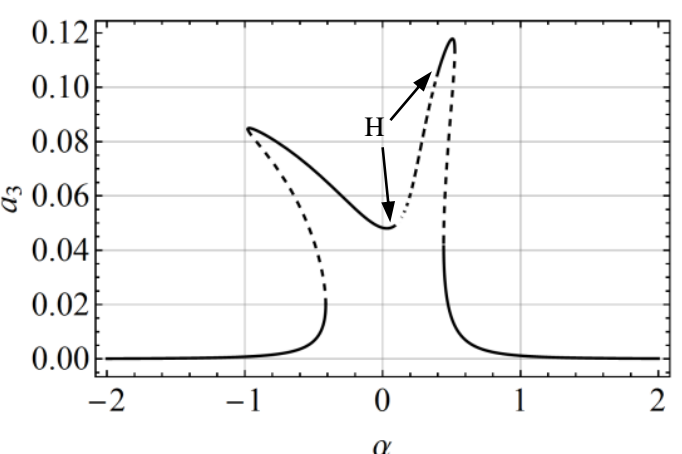

(b)

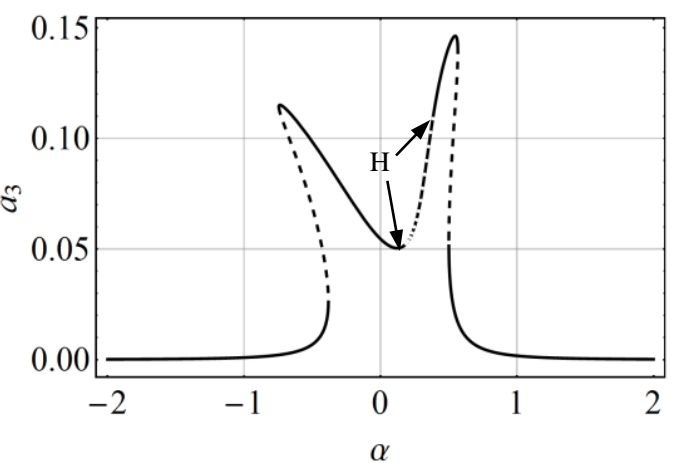

(d)

Figure 6. Frequency response curves of the first and third amplitudes as a function of the detuning parameter $\alpha$. (—) Stable fixed points, $(--)$ unstable fixed points. H denotes the point of a Hopf bifurcation. (a,b) $\theta=62^{\circ}, \mu_{1}=0.08, \mu_{2}=0.08, F_{m}=8$. (c,d) $\theta=32^{\circ}, \mu_{1}=0.08, \mu_{2}=0.08$ $F_{m}=23$.

As the frequency is swept from lower values, the response experiences a saddle node jump from the lower branch to the upper branch in the softening part of the response. Then, the system passes through forward and reverse subcritical Hopf bifurcations after which the system, due to internal resonance activation, experiences a hardening type response. This is apparent in both configuration cases. In addition, more forcing amplitude is required in case (b) to achieve a similar response observed in case (a) to compensate the influence of larger static deflection at lower configuration angles. In addition, the hardening type response in both cases is capped because the formulation accounts for the influence of cubic nonlinearities.

Furthermore, we examine the interaction by plotting the time histories and the corresponding phase spaces in Fig. 7 by substituting the amplitudes and corresponding phases in Eq. (32). 


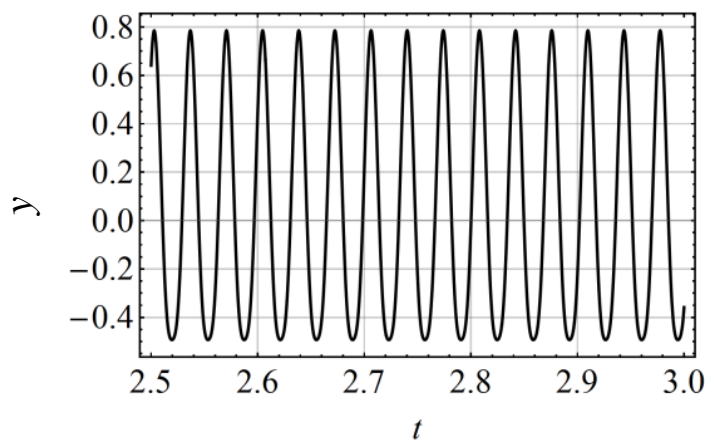

(a)

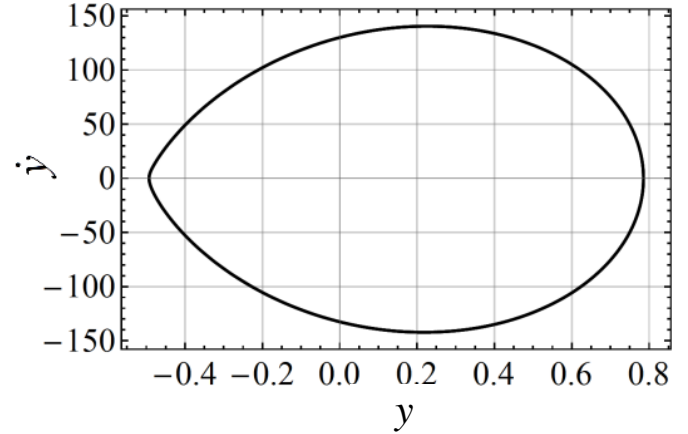

(b)

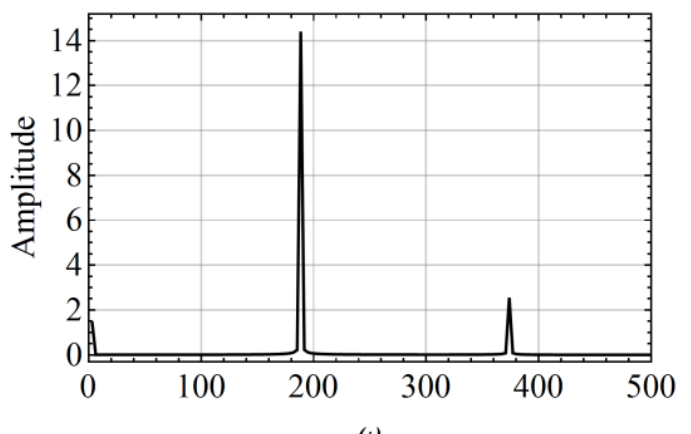

(c)

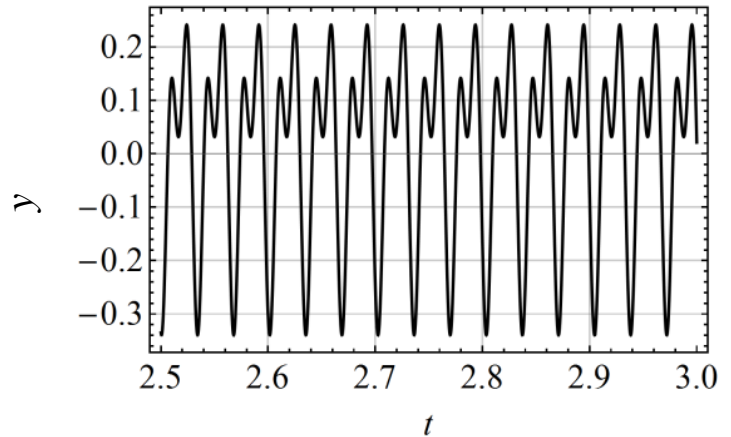

(d)

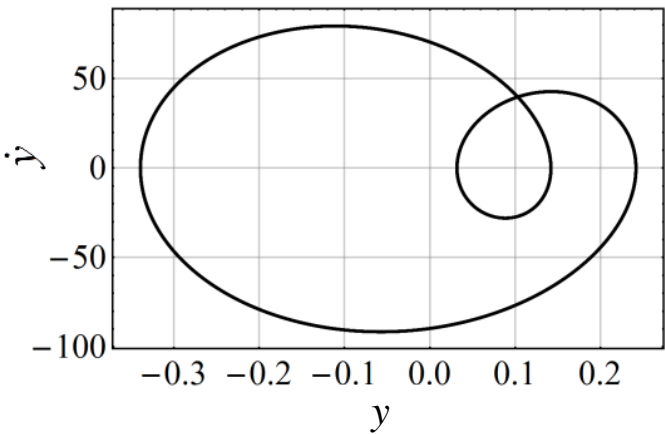

(e)



(f)

Figure 7. Response time history and their corresponding phase portraits for $\theta=62^{\circ}, \mu_{1}=0.08$, $\mu_{2}=0.08, F_{m}=8$. (a,b,c) $\alpha=-0.8$, (d,e,f) $\alpha=0.45$.

In Fig. 7a, the time history of these results was obtained from the lower branch of the softening response type at $\alpha=-0.8$. Away from the interaction zone, the response is dominated by a single period and confirmed by a closed loop in the 
phase plot Fig. 7b and the Fourier spectrum in Fig. 7c. In contrast, the plot in the interaction zone at $\alpha=0.45$ (Fig. 7d) shows a two-period response confirmed by the two loops in Fig. 7e and the competing amplitudes in the Fourier spectrum corresponding to each mode in Fig. 7f.

Moreover, the force response curve is obtained for both amplitudes by plotting the amplitudes against the excitation amplitude for difference values of $\alpha$. The results are depicted in Fig. 8.
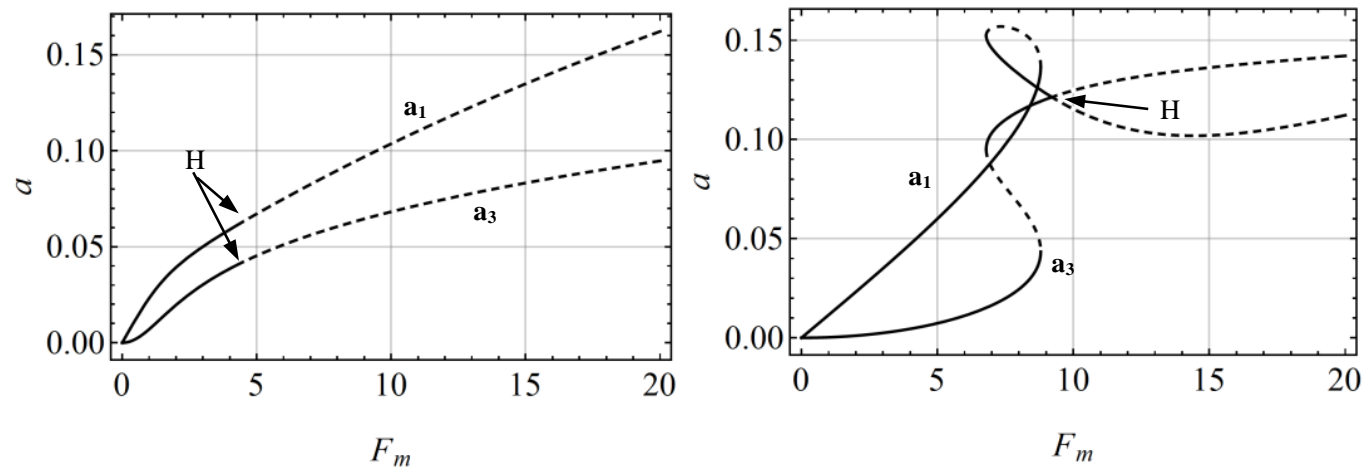

(a)

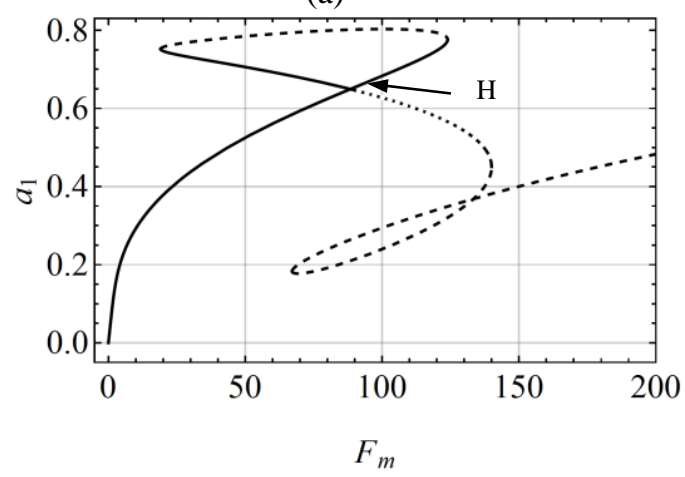

(c)

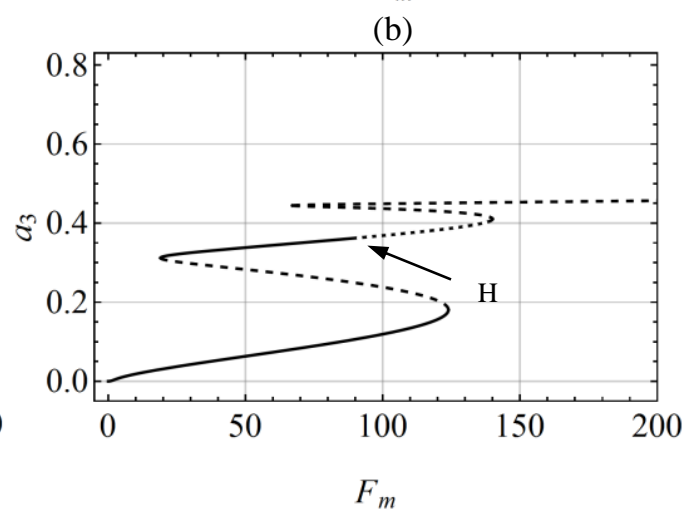

(d)

Figure 8. Force response curves of the first and third amplitudes as a function of forcing. $\theta=62^{\circ}$, $\mu_{1}=0.08, \mu_{2}=0.08$. (—) Stable fixed points, $(--)$ unstable fixed points. H denotes the point of a Hopf bifurcation. (a) $\alpha=0.20$, (b) $\alpha=0.45$, (c,d) $\alpha=0$. 
We plot the response in Fig. $8 \mathrm{a}$ and $8 \mathrm{~b}$ for small forcing values and large forcing values in Fig. 8c, 8d. Since the excitation is around the first natural frequency, the trivial solution is not present and the interaction is always present. As the forcing increases in Fig. 8a, the exchange of energy starts at very low forcing values promoting the interaction between the two modes while increasing the forcing further results into a Hopf bifurcation. In addition, in Fig. 8b we observe the emergence of a saddle node bifurcation of the softening band followed by a Hopf bifurcation when the forcing is increased. Extending the forcing range further in Fig. $8 \mathrm{c}, \mathrm{d}$ shows the occurrence of the saturation phenomena in the response of the third amplitude after Hopf bifurcation. These results suggest that care needs to be taken when designing a marine riser since the interaction can result into a bifurcation of the response even at very low external velocities.

To gain more insight on how the interaction varies with the applied tension, we vary the applied tension such that the third natural frequency is less than twice of the first natural frequency. Then, we increase the value of tension in a multistep process (solving for the static problem, linear eigenvalue problem, and then particular solutions) and solve the amplitude of each mode from Eq. (31). The detailed solutions of the first and third mode amplitudes corresponding to each tension value are available in the supplementary materials. The results at $\alpha=0$ (softening regime) and $\alpha=0.25$ (hardening regime) are shown in Fig. 9.

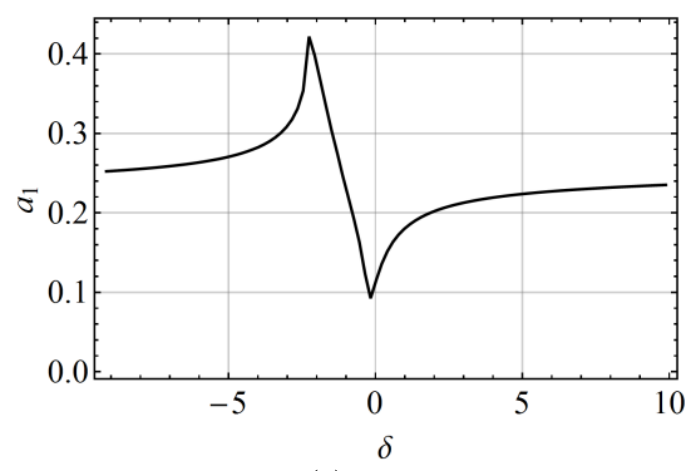

(a)

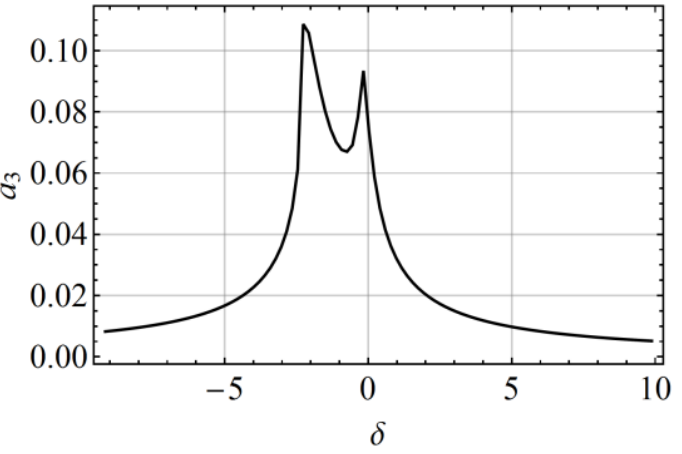

(b) 




(c)

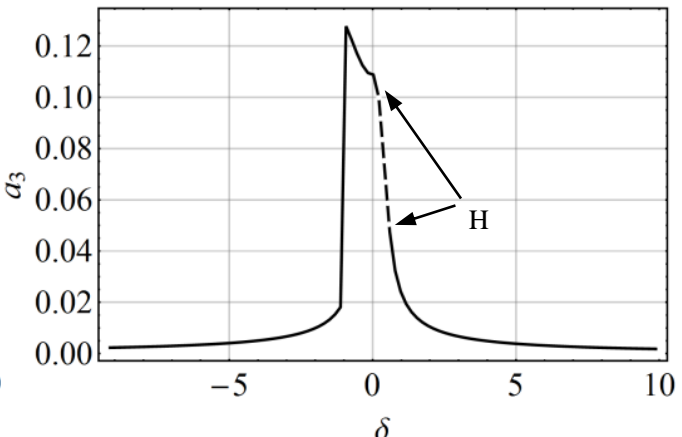

(d)

Figure 9. Frequency response curves showing the response of the first and third amplitude as a function of the detuning parameter $\delta . \theta=62^{\circ}, \mu_{1}=0.08, \mu_{2}=0.08, \alpha=0, F_{m}=8$. (—) Stable fixed solutions, $(--)$ unstable fixed solution. $\mathrm{H}$ denotes the point of a Hopf bifurcation. (a,b) $\alpha=0$, (c,d) $\alpha=0.25$.

As noted from Fig. 9a, away from the interaction zone, at $\delta=-8$, the energy exchange due to the internal resonance activation is not present because the frequency ratio is not exactly two, $\omega_{3} / \omega_{1} \approx 1.95$. Increasing the tension further brings the ratio closer to two where we notice the first sign of the interaction in the peaks. As the tension is increased, the first mode amplitude decreases and the third natural frequency tends to decrease then increase as a result of the energy exchange. Then, increasing the tension further results in a ratio that is greater than two where the interaction decreases until it diminishes away. Similarly, in Fig. 9b, we notice that the interaction results into activation of the softening band at higher values of $\delta$ compared to Fig. 9a. This implies that early signs of interaction appear first at a lower frequency ratio in the hardening before the softening appears. In addition, a Hopf bifurcation is observed when increasing the tension further because of such interaction.

To further investigate the dynamics in between the two Hopf bifurcation points, we integrate in time the modulation equations, Eqs. (31). It is well known that a limit cycle solution is born whenever a Hopf bifurcation occurs [39] and this turns the fixed points of the system to unstable solution near the bifurcation points. Therefore, we plot the response of the riser and compare it with that 
obtained by numerically integrating the reduced order model from the Galerkin solution. The results are depicted in Fig. 10 showing good agreement among the results.

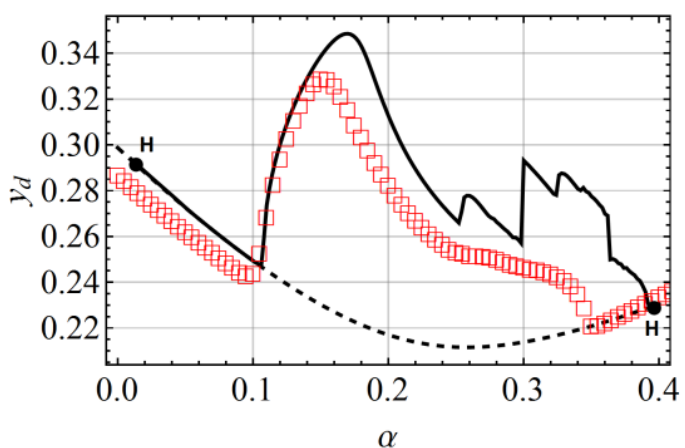

(a)

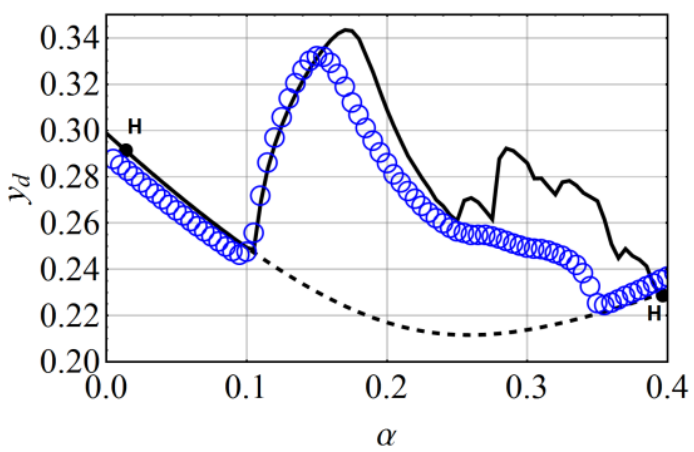

(b)

Figure 10. Frequency response curves showing the response of the riser between the Hopf bifurcation points of Fig. 5. $\theta=62^{\circ}, \mu_{1}=0.08, \mu_{2}=0.08, F_{m}=8$. (—) Solutions obtained by integrating Eqs. (31), ( - - ) unstable fixed point solutions, $(\square, \bigcirc)$ solutions obtained by numerically integrating the Galerkin solution. H denotes the point of a Hopf bifurcation. (a) Forward sweep, (b) backward sweep.

One can observe some differences between the forward and backward sweep results from MTS indicating multi coexisting states. This can be attributed to the presence of complex dynamics, potentially chaotic, which has been reported in the Hopf bifurcation regime in similar systems undergoing a two-one internal resonance [13].

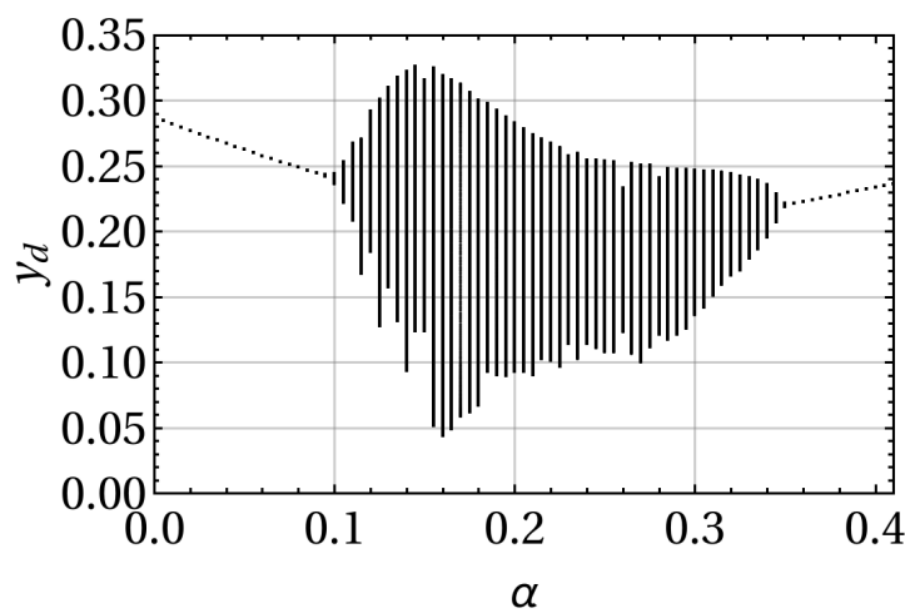

Figure 11. Bifurcation diagram of the riser's deflection around the first primary resonance by plotting the Poincare displacements for each value of $\alpha . \theta=62^{\circ}, \mu_{1}=0.08, \mu_{2}=0.08, F_{m}=8$. 
A bifurcation diagram corresponding to the Galerkin solution is shown in Fig. 11. The solution after the Hopf bifurcation between $\alpha=0.02$ and $\alpha=0.1$ exhibits a periodic solution represented by discrete points in the Poincare diagram and confirmed by the time histories and phase portrait plots, as shown for example in Fig. 12a and 12b. As we sweep closer to $\alpha=0.1$, a transition in the response occurs as observed from the shift of the harmonic peak in the time history Fig. 12c and the deformed phase portrait, Fig. 12d. Further analysis for the dynamic solutions near the Hopf bifurcation can be done based on continuation algorithms combined with the Floquet theory.

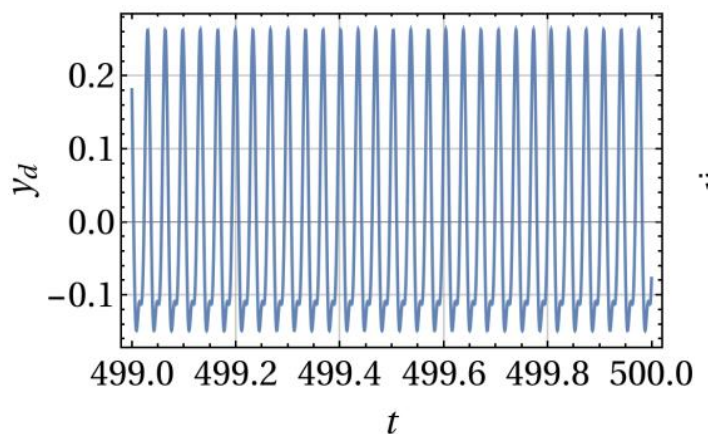

(a)

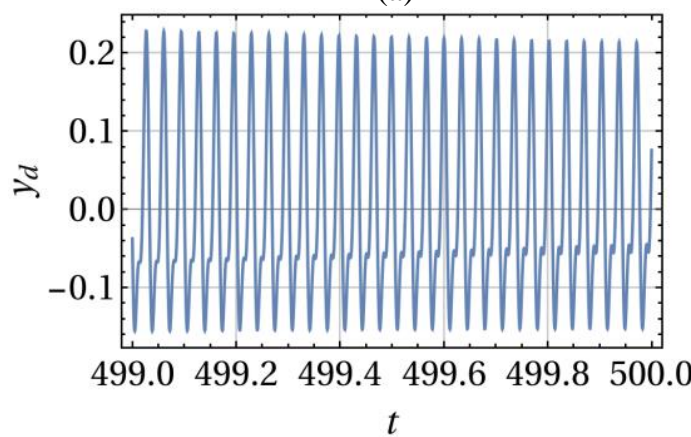

(c)

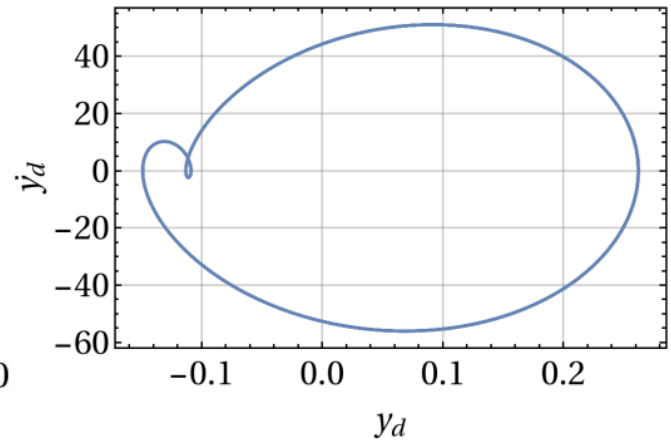

(b)



(d)

Figure 12. Time histories and their corresponding phase portraits at different values of $\alpha$. $\theta=62^{\circ}, \mu_{1}=0.08, \mu_{2}=0.08, F_{m}=8$. (a,b) $\alpha=0.05$, (c,d) $\alpha=0.115$

To further analyze the type of the response after the transition regime, the time history, the Poincare diagram, and the power spectrum are plotted for $\alpha=0.15$ in Fig. 13 . 


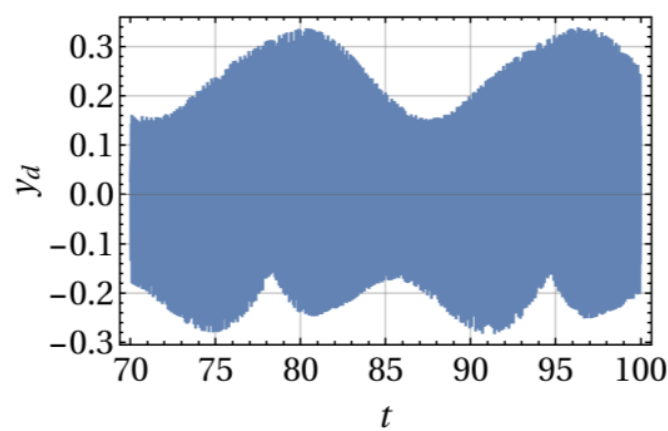

(a)



(b)

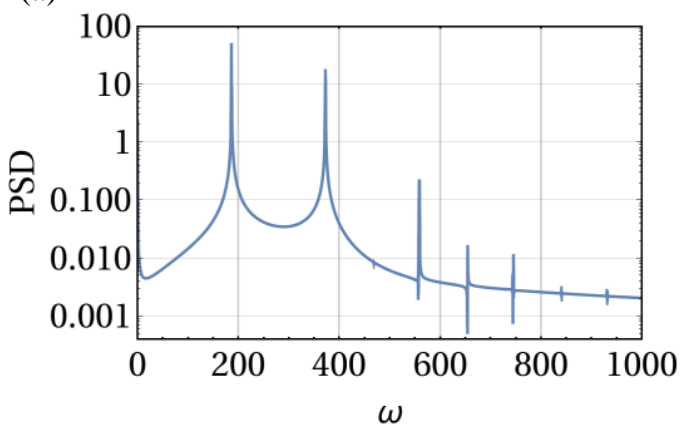

(c)

Figure 13. (a) Time history and the corresponding (b) Poincare section and (c) power spectra based on Galerkin method for for $\alpha=0.15 \theta=62^{\circ}, \mu_{1}=0.08, \mu_{2}=0.08, F_{m}=8$.

The results shown in Fig. 13 indicate that the solutions corresponding to $\alpha=0.15$ show a quasi-periodic motion. This is confirmed by the presence of the closed loop in the Poincare section in Fig. 13b and the multiple harmonics that appear in the power spectral density of the response. The influence of two-to-one internal resonance on the response is realized by plotting the temporal modal coefficients corresponding to the first and third modes from the Galerkin solution in Fig. 14. The coefficients of other participating modes are neglected from the analysis because their amplitude is very low, as clarified in Appendix C.
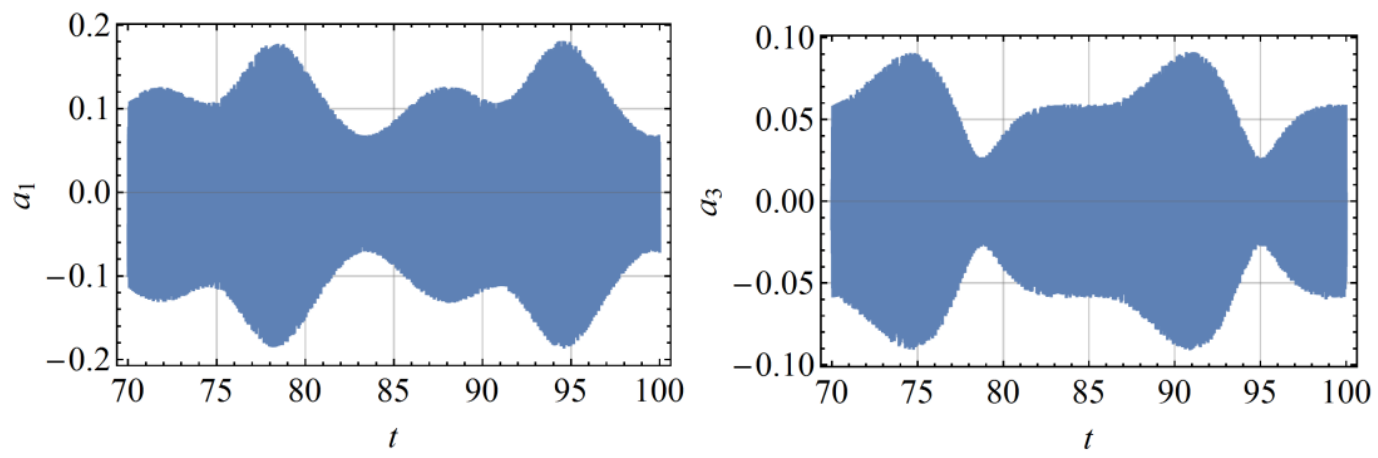
(a)

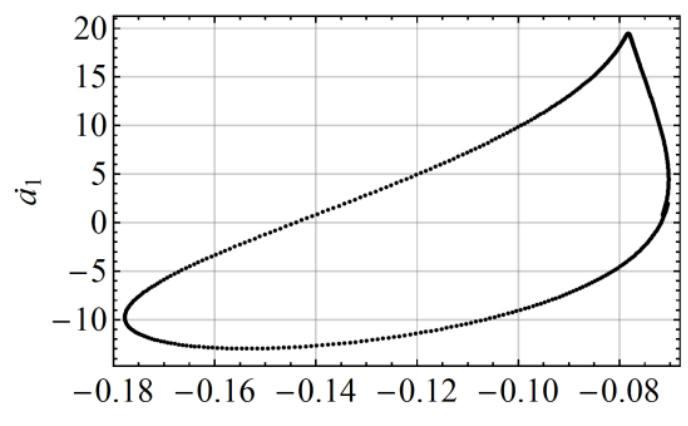

$a_{1}$

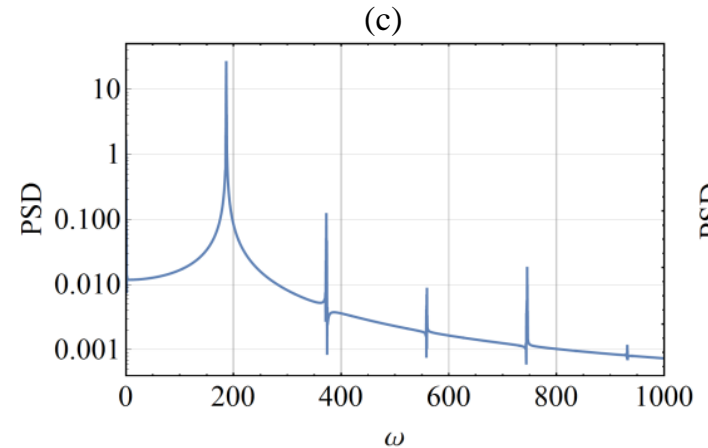

(e) (b)

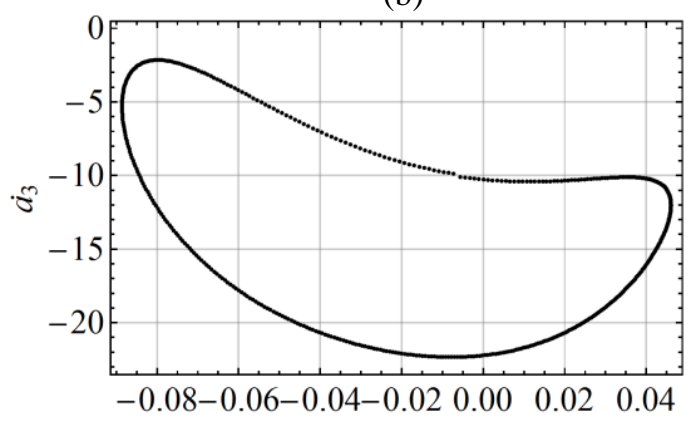

$a_{3}$

(d)

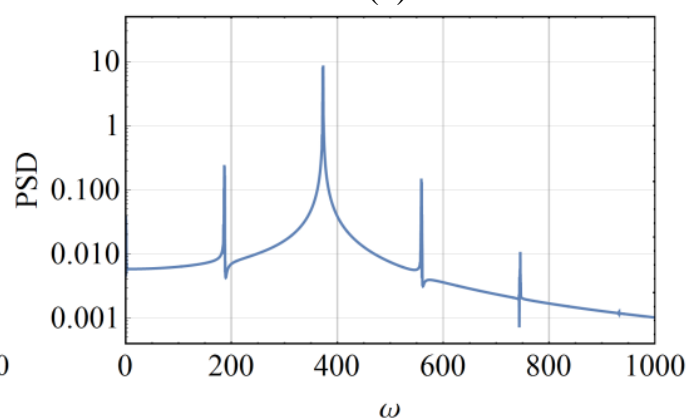

(f)

Figure 14. (a) (b) Time histories of $a_{1}$ and $a_{3}$ using the Galerkin method. (c) (d) Poincaré sections

(e) (f) Power spectra corresponding to $a_{1}$ and $a_{3}$, respectively. $\theta=62^{\circ}, \mu_{1}=0.08, \mu_{2}=0.08$, $\alpha=0.15, \bar{F}_{m}=8$.

It is noticeable from the time histories in Fig. 14a and Fig. 14b that the response is quasi-periodic. This is confirmed by the closed loop observed in the Poincare sections in Fig. 14c and Fig 14d. In addition, the power spectral density functions show that new frequencies start to appear apart from the first and third natural frequencies. This can lead to chaotic behavior and can be detrimental to the lifetime of the riser structure. Lastly, we compare the solution obtained by integrating the modulation equations with the previous results obtained by Galerkin and we plot the time history, the Poincare section, and power spectrum in Fig. 15. 


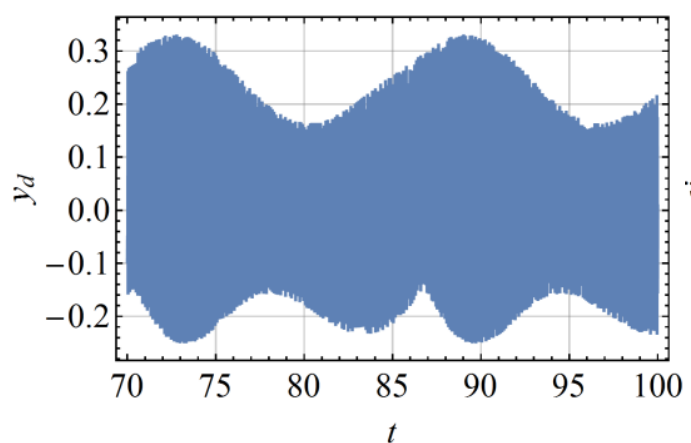

(a)

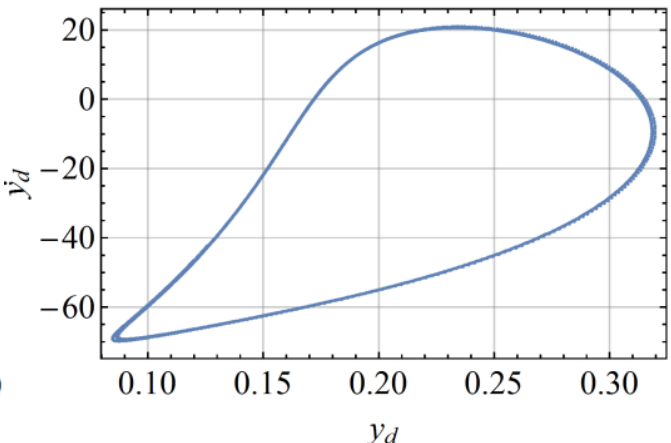

(b)

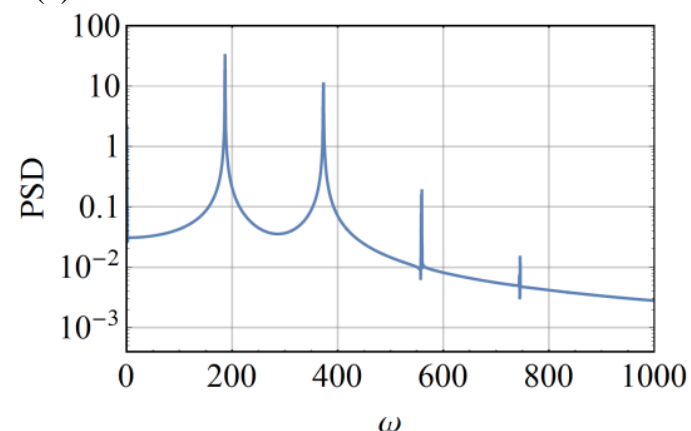

(c)

Figure 15. (a) Time history and the corresponding (b) Poincare section and (c) power spectra based on MTS method for $\alpha=0.15 \theta=62^{\circ}, \mu_{1}=0.08, \mu_{2}=0.08, F_{m}=8$.

The time history results from Fig. 15a show good agreement with that shown in Fig. 13a, based on the Galerkin solution. Similar agreement is observed in predicting the quasi-periodic closed-loop Poincare section in Fig. 15b when compared to that of Fig. 13b. Moreover, a difference is noted when examining the power spectrum of the MTS solution Fig. 15c compared to that of Fig. 13c. This is attributed to the influence of the other modes shapes not captured in the MTS and higher order corrections.

\section{Conclusions}

In this paper, the two-to-one internal resonance of an inclined riser has been studied. We used the method of multiple scales to investigate the interaction between the first and third natural frequencies when their frequency ratio gets close to 2 . The results show good agreement between the multiple scales solution and the Galerkin solution when the internal resonance is activated. A further 
investigation has been carried out by plotting the frequency response and force response curves showing the nature of the internal resonance interaction. Also, the system show bifurcation from a stable state to a limit cycle through Hopf bifurcation, which can lead to chaotic behavior that hinders the life time riser structure undergoing these oscillations. A future prospective from this work is realized in studying the full VIV interaction undergoing internal resonance interaction via a Van Der Pol oscillator using multiple scales theory and shooting method

\section{Acknowledgements}

We acknowledge the financial support of King Abdullah Univeristy of Science and Technology and Saudi Aramco.

\section{Conflict of interest}

The authors declare that they have no conflict of interest.

\section{Supplementary Material}

Supplementary video 1 (avi $48.4 \mathrm{MB}$ )

Supplementary video 2 (avi 48.4 MB)

Supplementary information 1 (pdf $165 \mathrm{kB})$

\section{Appendix A: Self-adjoint proof}

In this appendix, we demonstrate by virtue of using integration by parts that the solution to the linear Eq. (11) is self-adjoint. Due to the internal resonance interaction, the solution to be utilized consists of two modes, namely, $\phi_{m}(x) e^{ \pm i \omega_{m} T_{0}}$ and $\phi_{n}(x) e^{ \pm i \omega_{n} T_{0}}$ corresponding to modes $m$ and $n$, respectively. To verify that the problem is self-adjoint, we substitute the solutions from both modes in Eq. (11), and multiply the equation with $\phi_{j}(x) e^{ \pm i \omega_{j} T_{0}}$, then integrate by parts from $x=0$ to $x=1$ to obtain 


$$
\begin{aligned}
& {\left[\phi_{j} \phi_{m}^{\prime \prime \prime}-\phi_{j}^{\prime} \phi_{m}^{\prime \prime}+\phi_{j}^{\prime \prime} \phi_{m}{ }^{\prime}-\phi_{j}^{\prime \prime \prime} \phi_{m}+\bar{T}_{s} \phi_{j} \phi_{m}{ }^{\prime}+\overline{T_{s}} \phi_{j}^{\prime} \phi_{m}-\bar{\sigma} x \phi_{j} \phi_{m}{ }^{\prime}+\bar{\sigma}\left(x \phi_{j}\right)^{\prime} \phi_{m}-\bar{\sigma} \phi_{j} \phi_{m}\right]_{0}^{1}} \\
& {\left[\phi_{j} \phi_{n}^{\prime \prime \prime}-\phi_{j}^{\prime} \phi_{n}^{\prime \prime}+\phi_{j}^{\prime \prime} \phi_{n}^{\prime}-\phi_{j}^{\prime \prime \prime} \phi_{n}+\bar{T}_{s} \phi_{j} \phi_{n}^{\prime}+\bar{T}_{s} \phi_{j}^{\prime} \phi_{n}-\bar{\sigma} x \phi_{j} \phi_{n}^{\prime}+\bar{\sigma}\left(x \phi_{j}\right)^{\prime} \phi_{n}-\bar{\sigma} \phi_{j} \phi_{n}\right]_{0}^{1}} \\
& +2 \eta\left[\phi_{j} y_{s}^{\prime \prime}\left(\int_{0}^{1} y_{s}^{\prime} \phi_{m}{ }^{\prime} \mathrm{d} x\right)+\phi_{m} y_{s}^{\prime \prime}\left(\int_{0}^{1} y_{s}^{\prime} \phi_{j}^{\prime} \mathrm{d} x\right)\right]_{0}^{1}+2 \eta\left[\phi_{j} y_{s}^{\prime \prime}\left(\int_{0}^{1} y_{s}^{\prime} \phi_{n}{ }^{\prime} \mathrm{d} x\right)+\phi_{n} y_{s}^{\prime}\left(\int_{0}^{1} y_{s}^{\prime} \phi_{j}^{\prime} \mathrm{d} x\right)\right]_{0}^{1} \\
& \int_{0}^{1} \phi_{m}\left[-\omega_{m}^{2} \phi_{j}+\phi_{j}^{i v}+\overline{T_{s}} \phi_{j}^{\prime \prime}-\bar{\sigma}\left(x \phi_{j}\right)^{\prime \prime}+\bar{\sigma} \phi_{j}^{\prime}-2 \eta y_{s}^{\prime \prime}\left(\int_{0}^{1} y_{s}^{\prime}(x) \phi_{j}^{\prime} \mathrm{d} x\right)\right] \mathrm{d} x \\
& \int_{0}^{1} \phi_{n}\left[-\omega_{n}^{2} \phi_{j}+\phi_{j}^{i v}+\bar{T}_{s} \phi_{j}^{\prime \prime}-\bar{\sigma}\left(x \phi_{j}\right)^{\prime \prime}+\bar{\sigma} \phi_{j}^{\prime}-2 \eta y_{s}^{\prime \prime}\left(\int_{0}^{1} y_{s}^{\prime}(x) \phi_{j}^{\prime} \mathrm{d} x\right)\right] \mathrm{d} x
\end{aligned}
$$

We observe from Eq. (A.1) that the solution $\phi_{j}(x) e^{ \pm i \omega_{j} T_{0}}$ satisfies the eigenvalue problem Eq. (8) and it is self-adjoint.

\section{Appendix B: Definition of third order solvability condition coupling}

\section{coefficients}

In this appendix, we provide the definition of the coupling coefficients $K_{1}$, $K_{2}, K_{3}$ and $K_{4}$ pertaining to Eq. (23) and Eq. (24) given by

$$
\begin{aligned}
K_{1} & =2 \eta \int_{0}^{1}\left(\int_{0}^{1} \phi_{n}^{\prime} \psi_{1}^{\prime} \mathrm{d} x\right) \phi_{m} y_{s}^{\prime \prime} \mathrm{d} x+2 \eta \int_{0}^{1}\left(\int_{0}^{1} \phi_{m}^{\prime} \psi_{4}^{\prime} \mathrm{d} x\right) \phi_{m} y_{s}^{\prime \prime} \mathrm{d} x+2 \eta \int_{0}^{1}\left(\int_{0}^{1}\left(\phi_{n}^{\prime}\right)^{2} \mathrm{~d} x\right) \phi_{m} \phi_{m} \mathrm{~d}^{\prime} \mathrm{d} \\
& +2 \eta \int_{0}^{1}\left(\int_{0}^{1} y_{s}^{\prime} \psi_{4}^{\prime} \mathrm{d} x\right) \phi_{m} \phi_{m}^{\prime \prime} \mathrm{d} x+4 \eta \int_{0}^{1}\left(\int_{0}^{1} \phi_{m}^{\prime} \phi_{n}^{\prime} \mathrm{d} x\right) \phi_{m} \phi_{n}^{\prime \prime} \mathrm{d} x+2 \eta \int_{0}^{1}\left(\int_{0}^{1} y_{s}^{\prime} \psi_{1}^{\prime} \mathrm{d} x\right) \phi_{m} \phi_{n}^{\prime \prime} \mathrm{d} x \\
& +2 \eta \int_{0}^{1}\left(\int_{0}^{1} y_{s}^{\prime} \phi_{n}^{\prime} \mathrm{d} x\right) \phi_{m} \psi_{1}^{\prime \prime} \mathrm{d} x+2 \eta \int_{0}^{1}\left(\int_{0}^{1} y_{s}^{\prime} \phi_{m}^{\prime} \mathrm{d} x\right) \phi_{m} \psi_{4}^{\prime \prime} \mathrm{d} x \\
K_{2}= & 2 \eta \int_{0}^{1}\left(\int_{0}^{1} \phi_{m}^{\prime} \psi_{3}^{\prime} \mathrm{d} x\right) \phi_{m} y_{s}^{\prime \prime} \mathrm{d} x+3 \eta \int_{0}^{1}\left(\int_{0}^{1}\left(\phi_{m}^{\prime}\right)^{2} \mathrm{~d} x\right) \phi_{m} \phi_{m}^{\prime \prime} \mathrm{d} x+2 \eta \int_{0}^{1}\left(\int_{0}^{1} y_{s}^{\prime} \psi_{3}^{\prime} \mathrm{d} x\right) \phi_{m} \phi_{m}^{\prime \prime} \mathrm{d} x \\
& +2 \eta \int_{0}^{1}\left(\int_{0}^{1} y_{s}^{\prime} \phi_{m}^{\prime} \mathrm{d} x\right) \phi_{m} \psi_{3}^{\prime \prime} \mathrm{d} x \\
K_{3}= & 2 \eta \int_{0}^{1}\left(\int_{0}^{1} \phi_{m}^{\prime} \psi_{1}^{\prime} \mathrm{d} x\right) \phi_{n} y_{s}^{\prime \prime} \mathrm{d} x+2 \eta \int_{0}^{1}\left(\int_{0}^{1} \phi_{n}^{\prime} \psi_{3}^{\prime} \mathrm{d} x\right) \phi_{n} y_{s}^{\prime \prime} \mathrm{d} x+4 \eta \int_{0}^{1}\left(\int_{0}^{1} \phi_{m}^{\prime} \phi_{n}^{\prime} \mathrm{d} x\right) \phi_{n} \phi_{m}^{\prime \prime} \mathrm{d} x \\
& +2 \eta \int_{0}^{1}\left(\int_{0}^{1} y_{s}^{\prime} \psi_{1}^{\prime} \mathrm{d} x\right) \phi_{n} \phi_{m}^{\prime \prime} \mathrm{d} x+2 \eta \int_{0}^{1}\left(\int_{0}^{1}\left(\phi_{m}^{\prime}\right)^{2} \mathrm{~d} x\right) \phi_{n} \phi_{n}^{\prime \prime} \mathrm{d} x+2 \eta \int_{0}^{1}\left(\int_{0}^{1} y_{s}^{\prime} \psi_{3}^{\prime} \mathrm{d} x\right) \phi_{n} \phi_{n}^{\prime \prime} \mathrm{d} x \\
& +2 \eta \int_{0}^{1}\left(\int_{0}^{1} y_{s}^{\prime} \phi_{m}^{\prime} \mathrm{d} x\right) \phi_{n} \psi_{1}^{\prime \prime} \mathrm{d} x+2 \eta \int_{0}^{1}\left(\int_{0}^{1} y_{s}^{\prime} \phi_{n}^{\prime} \mathrm{d} x\right) \phi_{n} \psi_{3}^{\prime \prime} \mathrm{d} x
\end{aligned}
$$




$$
\begin{aligned}
K_{4} & =2 \eta \int_{0}^{1}\left(\int_{0}^{1} \phi_{n}^{\prime} \psi_{2}^{\prime} \mathrm{d} x\right) \phi_{n} y_{s}^{\prime \prime} \mathrm{d} x+2 \eta \int_{0}^{1}\left(\int_{0}^{1} \phi_{n}^{\prime} \psi_{4}^{\prime} \mathrm{d} x\right) \phi_{n} y_{s}^{\prime \prime} \mathrm{d} x+3 \eta \int_{0}^{1}\left(\int_{0}^{1}\left(\phi_{n}^{\prime}\right)^{2} \mathrm{~d} x\right) \phi_{n} \phi_{n}^{\prime \prime} \mathrm{d} x \\
& +2 \eta \int_{0}^{1}\left(\int_{0}^{1} y_{s}^{\prime} \psi_{2}^{\prime} \mathrm{d} x\right) \phi_{n} \phi_{n}^{\prime \prime} \mathrm{d} x+2 \eta \int_{0}^{1}\left(\int_{0}^{1} y_{s}^{\prime} \psi_{4}^{\prime} \mathrm{d} x\right) \phi_{n} \phi_{n}^{\prime \prime} \mathrm{d} x+2 \eta \int_{0}^{1}\left(\int_{0}^{1} y_{s}^{\prime} \phi_{n}^{\prime} \mathrm{d} x\right) \phi_{n} \psi_{2}^{\prime \prime} \mathrm{d} x \\
& +2 \eta \int_{0}^{1}\left(\int_{0}^{1} y_{s}^{\prime} \phi_{n}^{\prime} \mathrm{d} x\right) \phi_{n} \psi_{4}^{\prime \prime} \mathrm{d} x
\end{aligned}
$$

\section{Appendix C: Time History of Modal coefficients pertaining to the Galerkin}

\section{solution}

In this appendix, we plot the modal coefficients corresponding to the riser solution in Fig. 13, which has weak or negligible contribution.

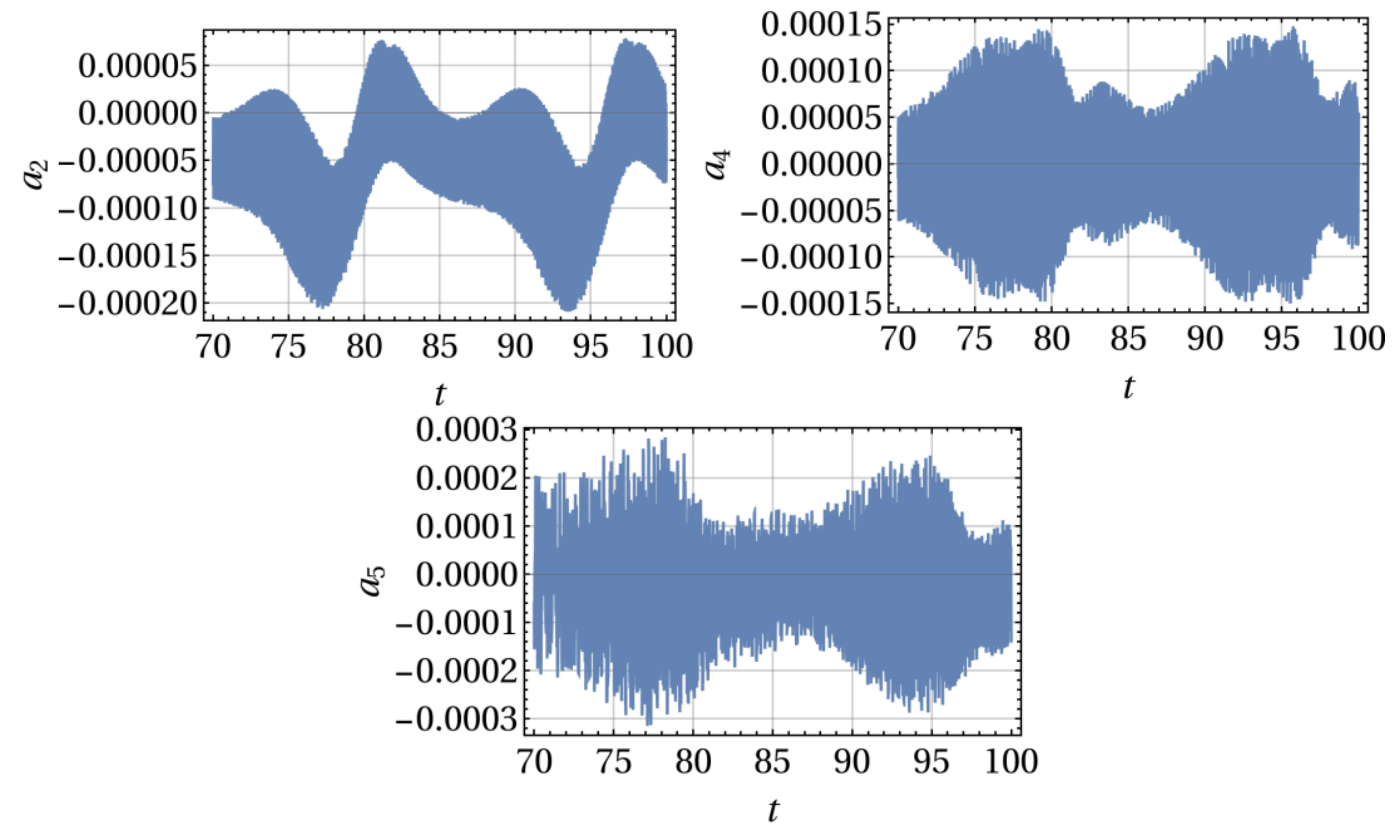

Figure C.1. Time histories of $a_{2}, a_{4}$ and $a_{5}$ modal coefficients from the Galerkin solution.

\section{References}

1. Païdoussis, M.P., Price, S.J., De Langre, E.: Fluid-structure interactions: crossflow-induced instabilities. Cambridge University Press, (2010)

2. Lacarbonara, W., Arafat, H.N., Nayfeh, A.H.: Non-linear interactions in imperfect beams at veering. International Journal of Non-Linear Mechanics 40(7), 987-1003 (2005). doi:https://doi.org/10.1016/j.ijnonlinmec.2004.10.006

3. Öz, H.R., Pakdemirli, M.: Two-to-one internal resonances in a shallow curved beam resting on an elastic foundation. Acta Mech. 185(3), 245-260 (2006). doi:https://doi.org/10.1007/s00707-006-0352-5

4. Nayfeh, A.H., Lacarbonara, W., Chin, C.-M.: Nonlinear Normal Modes of Buckled Beams: Three-to-One and One-to-One Internal Resonances.

Nonlinear Dyn. 18(3), 253-273 (1999). doi:https://doi.org/10.1023/A:1008389024738 
5. Chin, C., Nayfeh, A., Lacarbonara, W.: Two-to-one internal resonances in parametrically excited buckled beams. Proceedings of the 38th

AIAA/ASME/ASCE/AHS/ASC Structures, Structural Dynamics, and Materials, 7-10 (1997).

6. Chin, C.-M., Nayfeh, A.H.: Three-to-one internal resonances in hingedclamped beams. Nonlinear Dyn. 12(2), 129-154 (1997). doi:https://doi.org/10.1023/A:1008229503164

7. Benedettini, F., Rega, G., Vestroni, F.: Modal coupling in the free nonplanar finite motion of an elastic cable. Meccanica 21(1), 38-46 (1986). doi:https://doi.org/10.1007/bf01556315

8. Perkins, N.C.: Modal interactions in the non-linear response of elastic cables under parametric/external excitation. International Journal of Non-Linear Mechanics 27(2), 233-250 (1992). doi:https://doi.org/10.1016/00207462(92)90083-J

9. Gattulli, V., Lepidi, M., Macdonald, J.H.G., Taylor, C.A.: One-to-two globallocal interaction in a cable-stayed beam observed through analytical, finite element and experimental models. International Journal of Non-Linear Mechanics 40(4), 571-588 (2005). doi:https://doi.org/10.1016/j.ijnonlinmec.2004.08.005

10. Srinil, N., Rega, G.: Two-to-one resonant multi-modal dynamics of horizontal/inclined cables. Part II: Internal resonance activation, reduced-order models and nonlinear normal modes. Nonlinear Dyn. 48(3), 253-274 (2007). doi:https://doi.org/10.1007/s11071-006-9087-z

11. Srinil, N., Rega, G., Chucheepsakul, S.: Two-to-one resonant multi-modal dynamics of horizontal/inclined cables. Part I: Theoretical formulation and model validation. Nonlinear Dyn. 48(3), 231-252 (2007). doi:https://doi.org/10.1007/s11071-006-9086-0

12. Mansour, A., Mekki, O.B., Montassar, S., Rega, G.: Catenary-induced geometric nonlinearity effects on cable linear vibrations. J. Sound Vibr. 413, 332-353 (2018). doi:https://doi.org/10.1016/j.jsv.2017.10.012

13. Nayfeh, A.H.: Nonlinear interactions: analytical, computational and experimental methods. Wiley, (2000)

14. Mazzilli, C.E., Sanches, C.T., Neto, O.G.B., Wiercigroch, M., Keber, M.: Non-linear modal analysis for beams subjected to axial loads: Analytical and finite-element solutions. International Journal of Non-Linear Mechanics 43(6), 551-561 (2008). doi:https://doi.org/10.1016/j.ijnonlinmec.2008.04.004

15. Tien, W.-M., Namachchivaya, N.S., Bajaj, A.K.: Non-linear dynamics of a shallow arch under periodic excitation - I.1:2 internal resonance. International Journal of Non-Linear Mechanics 29(3), 349-366 (1994). doi:https://doi.org/10.1016/0020-7462(94)90007-8

16. Xiong, L.-Y., Zhang, G.-C., Ding, H., Chen, L.-Q.: Nonlinear forced vibration of a viscoelastic buckled beam with 2: 1 internal resonance. Mathematical Problems in Engineering (2014). doi:http://dx.doi.org/10.1155/2014/906324

17. Yi, Z., Wang, L., Kang, H., Tu, G.: Modal interaction activations and nonlinear dynamic response of shallow arch with both ends vertically elastically constrained for two-to-one internal resonance. J. Sound Vibr. 333(21), 5511-5524 (2014). doi:https://doi.org/10.1016/j.jsv.2014.05.052

18. Ma, J., Gao, X., Liu, F.: Nonlinear lateral vibrations and two-to-one resonant responses of a single pile with soil-structure interaction. Meccanica 52(15), 3549-3562 (2017). doi:https://doi.org/10.1007/s11012-017-0681-6 
19. Sahoo, B., Panda, L., Pohit, G.: Stability, bifurcation and chaos of a traveling viscoelastic beam tuned to 3: 1 internal resonance and subjected to parametric excitation. International Journal of Bifurcation and Chaos 27(02), 1750017 (2017). doi:https://doi.org/10.1142/S0218127417500171

20. Yu, T.-J., Zhang, W., Yang, X.-D.: Global dynamics of an autoparametric beam structure. Nonlinear Dyn. 88(2), 1329-1343 (2017). doi:https://doi.org/10.1007/s11071-016-3313-0

21. Zhu, B., Dong, Y., Li, Y.: Nonlinear dynamics of a viscoelastic sandwich beam with parametric excitations and internal resonance. Nonlinear Dyn., 138 (2018). doi:https://doi.org/10.1007/s11071-018-4511-8

22. Neto, O.B., Mazzilli, C.: Evaluation of multi-modes for finite-element models: systems tuned into 1: 2 internal resonance. International journal of solids and structures 42(21), 5795-5820 (2005). doi:https://doi.org/10.1016/j.ijsolstr.2005.03.026

23. Srinil, N., Wiercigroch, M., O'Brien, P.: Reduced-order modelling of vortexinduced vibration of catenary riser. Ocean Eng. 36(17-18), 1404-1414 (2009). doi:https://doi.org/10.1016/j.oceaneng.2009.08.010

24. Srinil, N.: Multi-mode interactions in vortex-induced vibrations of flexible curved/straight structures with geometric nonlinearities. J. Fluids Struct. 26(78), 1098-1122 (2010). doi:http://dx.doi.org/10.1016/j.jfluidstructs.2010.08.005

25. Srinil, N.: Analysis and prediction of vortex-induced vibrations of variabletension vertical risers in linearly sheared currents. Appl. Ocean Res. 33(1), 4153 (2011). doi:http://dx.doi.org/10.1016/j.apor.2010.11.004

26. Chatjigeorgiou, I.K., Mavrakos, S.A.: Nonlinear resonances of parametrically excited risers - numerical and analytic investigation for $\Omega=2 \omega 1$. Comput. Struct. 83(8-9), 560-573 (2005). doi:http://dx.doi.org/10.1016/j.compstruc.2004.11.009

27. Franzini, G., Mazzilli, C.: Non-linear reduced-order model for parametric excitation analysis of an immersed vertical slender rod. International Journal of Non-Linear Mechanics 80, 29-39 (2016). doi:https://doi.org/10.1016/j.ijnonlinmec.2015.09.019

28. Zhang, Y.-L., Chen, L.-Q.: Steady-state response of pipes conveying pulsating fluid near a 2: 1 internal resonance in the supercritical regime. International Journal of Applied Mechanics 6(05), 1450056 (2014). doi:https://doi.org/10.1142/S1758825114500562

29. Wilson, J.F., Biggers, S.B.: Responses of Submerged, Inclined Pipelines Conveying Mass. Journal of Engineering for Industry 96(4), 1141-1146 (1974). doi:https://doi.org/10.1115/1.3438488

30. Alfosail, F.K., Nayfeh, A.H., Younis, M.I.: An analytic solution of the static problem of inclined risers conveying fluid. Meccanica 52(4), 1175-1187 (2016). doi:https://doi.org/10.1007/s11012-016-0459-2

31. Alfosail, F.K., Nayfeh, A.H., Younis, M.I.: Natural frequencies and mode shapes of statically deformed inclined risers. International Journal of NonLinear Mechanics 94, 12-19 (2017). doi:https://doi.org/10.1115/imece201666009

32. Khalak, A., Williamson, C.H.K.: Motions, Forces and Mode Transitions in Vortex-Induced Vibrations at Low Mass-Damping. J. Fluids Struct. 13(7), 813-851 (1999). doi:https://doi.org/10.1006/jfls.1999.0236

33. Facchinetti, M.L., de Langre, E., Biolley, F.: Coupling of structure and wake oscillators in vortex-induced vibrations. J. Fluids Struct. 19(2), 123-140 (2004). doi:http://dx.doi.org/10.1016/j.jfluidstructs.2003.12.004 
34. Nayfeh, A.H.: Resolving controversies in the application of the method of multiple scales and the generalized method of averaging. Nonlinear Dyn. 40(1), 61-102 (2005). doi:https://doi.org/10.1007/s11071-005-3937-y

35. Nayfeh, A.H.: Introduction to Perturbation Techniques. Wiley, (2011)

36. Cheney, E., Kincaid, D.: Numerical Mathematics and Computing. Cengage Learning, (2012)

37. Balachandran, B., Nayfeh, A.: Cyclic motions near a Hopf bifurcation of a four-dimensional system. Nonlinear Dyn. 3(1), 19-39 (1992). doi:https://doi.org/10.1007/BF00045469

38. Wolfram Research, I.: Mathematica. In, vol. Version 10.1. Wolfram Research, Inc., Champaign, Illinois, (2015)

39. Nayfeh, A.H., Mook, D.T.: Nonlinear oscillations. John Wiley \& Sons, (2008) 